\title{
SYNTHESIS AND MODIFICATION OF 2-[8-R $-\mathbf{R}-\mathbf{-} \mathbf{R}_{2}-10-\mathbf{R}_{3}-3-$ R-2-OXO-2H-[1,2,4]TRIAZINO[2,3-c]QUINAZOLINE-6-YL) THIO]ACETIC ACIDS AIMED AT SEARCHING EFFECTIVE SUBSTANCES WITH THE ANTIBACTERIAL AND ANTIFUNGAL ACTIVITY
}

\author{
I.S.Nosulenko, O.Yu.Voskoboynik, G.G.Berest, S.I.Kovalenko, \\ O.M.Kamyshnyi, N.M.Polishchuk \\ Zaporizhzhia State Medical University \\ Key words: synthesis; quinazolines; triazines; antibacterial action; antifungal action
}

\begin{abstract}
In the present paper 50 new derivatives of 2-[8- $R_{1}-9-R_{2}-10-R_{3}-3-R-2-0 x 0-2 H-[1,2,4]$ triazino[2,3-C] quinazoline-6-yl)thio]acetic acids have been described. It has been shown that alkylation of potassium 8- $R_{1}-9-R_{2}-10-R_{3}-3-R-2-o x o-2 H-[1,2,4]$ triazino[2,3-c]quinazolin-6-thiolates by chloracetic acid, chloracetamide, $N-R_{4}$-chloracetamides and chloracetonitrile yield the corresponding $2-\left[8-R_{1}-9-R_{2}-10-R_{3}-\right.$ 3-R-2-oxo-2H-[1,2,4]triazino[2, 3-c]quinazoline-6-yl)thio]acetic acids, their amides and nitriles. For the corresponding acids and nitriles the alternative synthetic approaches have been developed. Limitations of synthetic approaches concerning the synthesis of the target compounds have been also discussed. Thus, it has been shown that amides of 2-[8- $R_{1}-9-R_{2}-10-R_{3}-3-R-2-0 \times 0-2 H-[1,2,4]$ triazino[2,3-C] quinazoline-6-yl)thio]acetic acids derivatives can not be prepared by amonolysis of the corresponding ester due to the low reactivity of the compounds mentioned. It has been also stated that the synthesis of nitriles via dehydration of proper amides with phosphorous-oxychloride in dichlormethane was not successful in all cases. This fact was caused by low yields and problems with isolation of the target compounds from the reaction mixture. The structures of the compounds synthesized have been confirmed by ${ }^{1} \mathrm{H},{ }^{13} \mathrm{C} N M R, L C-M S$ analysis. The compounds synthesized have been tested for the antimicrobial and antifungal activity using standard test cultures: Staphylococcus aureus ATCC 25923, Escherichia coli ATCC 25922, Pseudomonas aeruginosa ATCC 27853 and Candida albicans ATCC 885-653. It has been shown that the compounds synthesized exhibit a high antimicrobial activity against St. aureus (compounds 3.3-3.6, 4.3-4.6, 4.7, 4.8, 4.13-4.16; MIC 12.5-25 $\mu \mathrm{g} / \mathrm{ml}$ ) and C. albicans (compounds 4.13, 4.14; MIC $12.5 \mu \mathrm{g} / \mathrm{ml})$. The "structure-activity" relationship has been discussed.
\end{abstract}

Quinazoline derivatives play an important role in the present list of medicines. First of all, the compounds mentioned are known as anticancer (afatinib, vandetanib, lapatinib, trimetrexate, gefitinib, raltitrexed), antifungal (albaconazol), antibacterial (nifurquinazol) drugs, etc. [3]. Some recent publications are devoted to the search of chemotherapeutics among condensed quinazolines, in particular benzimidazo[1,2-c]-, benzthiadiazolimidazo[1,2-c]-, triazolo[1,5-c]-, imidazo, [1,2,4]triazolo[ $[4,3-c]-$, triazino[ $[2,3-c]$ quinazolines $[1,2,4,5,7,8$, 10, 13-18]. Highly effective antimicrobial, antifungal, anticancer agents have been found among the compounds mentioned. Following to the strategy aimed at the directed search of biologically active compounds among S-substituted 3-R-6-thioxo-6,7-dihydro- $2 \mathrm{H}$ $[1,2,4]$ triazino[2,3-c]quinazoline-2-ones $[6-8,11,12]$, we were interested in chemical modification of the carboxylic group, aryl group in position 3 and introduction of halogen- and alkyl- substituents in positions 8, 9, 10 of the structure of the given compounds and evaluation of their antimicrobial and antifungal activity. The data obtained were used to understand the "structure-activity" relationship.

\section{Experimental Part}

\section{Chemistry}

1.1. General methods

Melting points were determined in open capillary tubes and were uncorrected. The elemental analyses $(\mathrm{C}, \mathrm{H}$, $\mathrm{N}, \mathrm{S}$ ) were performed using an ELEMENTAR vario EL Cube analyzer (USA). Analyses were indicated by the symbols of the elements or functions within $\pm 0.3 \%$ of the theoretical values. ${ }^{1} \mathrm{H}$ NMR spectra $(500 \mathrm{MHz})$ and ${ }^{13} \mathrm{C}$ NMR spectra $(100 \mathrm{MHz})$ were recorded on a Varian-Mercury 400 (Varian Inc., Palo Alto, CA, USA) spectrometer with TMS as an internal standard in DMSO- $d_{6}$ solution. LCMS were recorded using the chromatography/mass spectrometric system consisting of an "Agilent 1100 Series" high performance liquid chromatograph (Agilent, Palo Alto, CA, USA) equipped with a diode-matrix and an "Agilent LC/MSD SL" mass-selective detector (atmospheric pressure chemical ionization - APCI). Electron impact mass spectra (EI-MS) were recorded on a Varian $1200 \mathrm{~L}$ instrument at $70 \mathrm{eV}$ (Varian, USA). The purity of all compounds was checked by ${ }^{1} \mathrm{H}-\mathrm{NMR}$ and LC-MS.

Substances 1.1-1.19 and 2.1-2.19 were synthesized according to the procedures reported $[7,8]$. Other starting 
materials and solvents were obtained from commercially available sources and used without additional purification.

1.2. General procedure for the synthesis of $\left[\left(8-R_{1}-\right.\right.$ 9- $R_{2}-10-R_{3}-3-R-2-o x o-2 H-[1,2,4]$ triazino[2,3-c]quinazoline-6-yl)thiolacetic acids (3.1-3.14).

Method A. Add the solution of $0.94 \mathrm{~g}(10 \mathrm{mmol})$ chloracetic acid with $0.40 \mathrm{~g}(10 \mathrm{mmol})$ of sodium hydroxide in $5 \mathrm{ml}$ of water to the solution of potassium of the proper 8- $\mathrm{R}_{1}-9-\mathrm{R}_{2}-10-\mathrm{R}_{3}-3-\mathrm{R}-2$-oxo- $2 H$ - $[1,2,4]$ triazino[2,3-c] guinazoline-6-thiolates (2.1-2.18) $(10 \mathrm{mmol})$ in $20 \mathrm{ml}$ of water, reflux for 1.5 hours to the neutral $\mathrm{pH}$. Then add $50 \mathrm{ml}$ of water to the resulted mixture and filter. Acidify a filtrate with hydrochloric acid to $\mathrm{pH} 3$. Filter the solid obtained and dry. Recrystallize compounds from dioxane.

Method B. Add the proper 8- $\mathrm{R}_{1}-9-\mathrm{R}_{2}-10-\mathrm{R}_{3}-3-\mathrm{R}-6-$ thio-6,7-dihydro- $2 H$-[1,2,4]triazino[2,3-c]guinazoline2-ones (1.1-1.18) $(5 \mathrm{mmol})$ and $0.47 \mathrm{~g}(5 \mathrm{mmol})$ of chloracetic acid to the solution of $0.23 \mathrm{~g}(10 \mathrm{mmol})$ of metallic sodium in $20 \mathrm{ml}$ of ethanol, reflux for 1.5 hours to the neutral $\mathrm{pH}$. Then add $50 \mathrm{ml}$ of water to the resulted mixture and filter. Acidify a filtrate with hydrochloric acid to $\mathrm{pH} 3$. Filter the solid obtained and dry. Recrystallize compounds from dioxane.

2-[(3-Methyl-2-oxo-2H-[1,2,4]triazino[2,3-c]quinazoline-6-yl)thio]acetic acids (3.1). Yield-71.9\% (method A), 69.4\% (method B). M.p. $-238-240{ }^{\circ} \mathrm{C} ;{ }^{1} \mathrm{H}$ NMR: $\delta=2.36$ (s, $\left.3 \mathrm{H}, \mathrm{CH}_{3}\right), 4.06$ (s, 2H, -SCH $), 7.68-7.59$ (m, 2H, H-8, 10), 7.93 (t, 1H, $J=7.9$, H-9), 8.41 (d, 1H, $J=7.9, \mathrm{H}-11), 12.90$ (s, 1H, COOH); ${ }^{13} \mathrm{C}$ NMR: $\delta=18.19\left(\mathrm{CH}_{3}\right), 34.21\left(\mathrm{SCH}_{2}\right)$, 118.50 (11a), 126.00 (8), 126.76 (10), 128.01 (11), 136.02 (9), 144.09 (11b), 151.93 (3), 154.41 (6), 155.28 (7a), 160.98 (2), 170.04(COOH); LC-MS, $m / z=303[\mathrm{M}+1], 304$ [M+2]; Anal. Calcd for $\mathrm{C}_{13} \mathrm{H}_{10} \mathrm{~N}_{4} \mathrm{O}_{3} \mathrm{~S}$ : C, 51.65; H, 3.33; N, 18.53; S, 10.61; Found: C, 51.66; H, 3.34; N, 18.52; S, 10.61.

2-[(3-Phenyl-2-oxo-2H-[1,2,4]triazino[2,3-c]quinazoline-6-yl)thio]acetic acids (3.2). Yield-75.8\% (method A), $70.3 \%$ (method B). M.p. $-270-272{ }^{\circ} \mathrm{C} ;{ }^{1} \mathrm{H}$ NMR: $\delta=4.14$ (s, $\left.2 \mathrm{H},-\mathrm{SCH}_{2}\right), 7.66-7.58\left(\mathrm{~m}, 3 \mathrm{H}, \mathrm{H}-3^{\prime}, 4^{\prime}, 5^{\prime}\right), 7.74-7.68$ (m, 2H, $\mathrm{H}-8,10), 7.98$ (t, 1H, $J=7.9, \mathrm{H}-9), 8.28$ (d, 2H, $J=8.2, \mathrm{H}-2^{\prime}$, 6'), 8.49 (d, 1H, J=7.9, H-11), 12.97 (s, 1H, COOH); LC-MS, $m / z=307[\mathrm{M}+1], 309[\mathrm{M}+3]$; Anal. Calcd for $\mathrm{C}_{18} \mathrm{H}_{12} \mathrm{~N}_{4} \mathrm{O}_{3} \mathrm{~S}$ : C, 59.33; H, 3.32; N, 15.38; S, 8.80; Found: C, 59.33; H, 3.33; N, 15.37; S, 8.81.

2-[(3-(4'-Methylphenyl)-2-oxo-2H-[1,2,4]triazino [2,3-c]quinazoline-6-yl)thio]acetic acids (3.3). Yield - 84.9\% (method A) and 80.6\% (method B). M.p. $-234-236{ }^{\circ} \mathrm{C} ;{ }^{1} \mathrm{H}$ NMR: $\delta=2.38\left(\mathrm{~s}, 3 \mathrm{H}, \mathrm{CH}_{3}\right), 4.10\left(\mathrm{~s}, 2 \mathrm{H},-\mathrm{SCH}_{2}\right), 7.37(\mathrm{~d}, 2 \mathrm{H}, J$ $\left.=8.2, \mathrm{H}_{-} 3^{\prime}, 5^{\prime}\right), 7.71-7.61(\mathrm{~m}, 2 \mathrm{H}, \mathrm{H}-8,10), 7.94(\mathrm{t}, 1 \mathrm{H}, J=7.9$, H-9), 8.20 (d, 2H, $J=8.2$, H-2', 6'), 8.44 (d, 1H, $J=7.9$, H-11), 12.93 (s, $1 \mathrm{H}, \mathrm{COOH})$; LC-MS, $m / z=321\left[\mathrm{M}-\mathrm{CH}_{2} \mathrm{COOH}\right]^{+}$, $379[\mathrm{M}+1], 381[\mathrm{M}+3]$; Anal. Calcd for $\mathrm{C}_{19} \mathrm{H}_{14} \mathrm{~N}_{4} \mathrm{O}_{3} \mathrm{~S}: \mathrm{C}$, 60.31; H, 3.73; N, 14.81; S, 8.47; Found: C, 60.32; H, 3.73; N, $14.81 ; \mathrm{S}, 8.49$

2-[(3-(3',4'-Dimethylphenyl)-2-oxo-2H-[1,2,4]triazino [2,3-c]quinazoline-6-yl)thio]acetic acids (3.4). Yield-85.4\% $(\operatorname{method} A), 80.9 \%$ (method B). M.p. $-226-228^{\circ} \mathrm{C} ;{ }^{1} \mathrm{H}$ NMR: $\delta=2.31\left(\mathrm{~d}, 6 \mathrm{H}, J=4.1,3,4-\left(\mathrm{CH}_{3}\right)_{2}\right), 4.11\left(\mathrm{~s}, 2 \mathrm{H},-\mathrm{SCH}_{2}\right), 7.33$ (d, 1H, $J=8.1, \mathrm{H}^{-5}$ '), 7.72-7.64 (m, 2H, H-10, 8), 7.95 (t, 1H,
$J=7.9$, H-9), 8.04 (d, 1H, $J=8.1$, H-6'), 8.07 (s, 1H, H-2'), 8.46 (d, $1 \mathrm{H}, J=7.9, \mathrm{H}-11), 12.99$ (s, 1H, COOH); LC-MS, $m / z$ $=335\left[\mathrm{M}-\mathrm{CH}_{2} \mathrm{COOH}\right]^{+}, 393[\mathrm{M}+1], 395[\mathrm{M}+3]$; Anal. Calcd for $\mathrm{C}_{20} \mathrm{H}_{16} \mathrm{~N}_{4} \mathrm{O}_{3} \mathrm{~S}$ : C, 61.21; H, 4.11; N, 14.28; S, 8.17; Found: C, 61.23; H, 4.13; N, 14.29; S, 8.18.

2-[(3-(4'-Ethylphenyl)-2-oxo-2H-[1,2,4]triazino [2,3-c] quinazoline-6-yl)thio]acetic acids (3.5). Yield75.9\%. M.p. $-161-163^{\circ} \mathrm{C} ;{ }^{1} \mathrm{H}$ NMR $\delta: 1.31$ (t, $J=7.5 \mathrm{~Hz}, 3 \mathrm{H}$, $\left.\mathrm{CH}_{2} \mathrm{CH}_{3}\right), 2.77$ (q, $\left.J=7.3 \mathrm{~Hz}, 2 \mathrm{H}, \underline{\mathrm{CH}}_{2} \mathrm{CH}_{3}\right), 4.07$ (s, $2 \mathrm{H},-\mathrm{SCH}_{2}$ ), $7.37\left(\mathrm{~d}, J=8.2 \mathrm{~Hz}, 2 \mathrm{H}, 3-\mathrm{Ph} \mathrm{H}-3^{\prime}, 5^{\prime}\right), 7.65$ (t, $J=7.6 \mathrm{~Hz}, 1 \mathrm{H}$, $\mathrm{H}-10), 7.75$ (d, $J=8.0 \mathrm{~Hz}, 1 \mathrm{H}, \mathrm{H}-8), 7.93$ (t, $J=7.5 \mathrm{~Hz}, 1 \mathrm{H}$, H-9), 8.28 (d, $\left.J=8.0 \mathrm{~Hz}, 2 \mathrm{H}, 3-\mathrm{PhH}-2^{\prime}, 6^{\prime}\right), 8.53$ (d, $J=7.9 \mathrm{~Hz}$, 1H, H-11), 12.79 (s, 1H, COOH); LC-MS, m/z = 393 [M+1], $395[\mathrm{M}+3]$; Anal. calcd. for $\mathrm{C}_{20} \mathrm{H}_{1} 6 \mathrm{~N}_{4} \mathrm{O}_{3} \mathrm{~S}$ : C, 61.21; H, 4.11; N, 14.28; S, 8.17; Found: C, 61.24; H, 4.11; N, 14.27; S, 8.19.

2-[(3-(4'-i-Propylphenyl)-2-oxo-2H-[1,2,4]triazino [2,3-c] quinazoline-6-yl)thio] acetic acids (3.6). Yield-43.3\%. M.p. $-236-238^{\circ} \mathrm{C}$; ${ }^{1} \mathrm{HNMR} \delta: 1.32\left(\mathrm{~d}, J=6.8 \mathrm{~Hz}, 6 \mathrm{H}, \mathrm{CH}\left(\mathrm{CH}_{3}\right)_{2}\right)$, $3.02\left(\mathrm{dt}, J=13.3,6.5 \mathrm{~Hz}, 1 \mathrm{H}, \underline{\mathrm{CH}}\left(\mathrm{CH}_{3}\right)_{2}\right), 4.05\left(\mathrm{~s}, 2 \mathrm{H},-\mathrm{SCH}_{2}\right)$, 7.38 (d, $\left.J=8.1 \mathrm{~Hz}, 2 \mathrm{H}, 3-\mathrm{Ph} \mathrm{H}-3{ }^{\prime}, 5^{\prime}\right), 7.63$ (t, $J=7.5 \mathrm{~Hz}, 1 \mathrm{H}$, $\mathrm{H}-10), 7.75$ (d, $J=8.0 \mathrm{~Hz}, 1 \mathrm{H}, \mathrm{H}-8), 7.91$ (t, $J=7.5 \mathrm{~Hz}, 1 \mathrm{H}$, H-9), 8.29 (d, $\left.J=8.1 \mathrm{~Hz}, 2 \mathrm{H}, 3-\mathrm{Ph} \mathrm{H}-2^{\prime}, 6^{\prime}\right), 8.54$ (d, $J=7.9 \mathrm{~Hz}$, $1 \mathrm{H}, \mathrm{H}-11), 12.82$ (s, 1H, COOH); LC-MS, m/z = 407 [M+1], $409[\mathrm{M}+3]$; Anal. calcd. for $\mathrm{C}_{21} \mathrm{H}_{18} \mathrm{~N}_{4} \mathrm{O}_{3} \mathrm{~S}: \mathrm{C}, 62.05 ; \mathrm{H}, 4.46$; N, 13.78; S, 7.89; Found: C, 62.07; H, 4.45; N, 13.79; S, 7.88.

2-2[(3-(4'-Methoxyphenyl)-2-oxo-2H-[1,2,4] triazino [2,3-c]quinazoline-6-yl)thio]acetic acids (3.7). Yield-77.4\% (method A), 70.3\% (method B). M.p. $-238-242^{\circ} \mathrm{C} ;{ }^{1} \mathrm{H}$ NMR: $\delta=3.84\left(\mathrm{~s}, 3 \mathrm{H}, \mathrm{OCH}_{3}\right), 4.10\left(\mathrm{~s}, 2 \mathrm{H},-\mathrm{SCH}_{2}\right), 7.11(\mathrm{~d}, 2 \mathrm{H}, J=8.8$, H-3', 5'), 7.72-7.60 (m, 2H, H-10, 8), 7.93 (t, 1H, J=7.9, H-9), 8.34 (d, 2H, J=8.8, H-2', 6'), 8.44 (d, 1H, J=7.9, H-11), 12.89 (s, 1H, COOH); ${ }^{13} \mathrm{C}$ NMR: $\delta=34.22\left(\mathrm{SCH}_{2}\right), 55.94\left(\mathrm{OCH}_{3}\right)$, 114.45 (3',5'-Ph), 118.21 (11a), 124.27 (8), 126.00 (10), 126.84 (1'-Ph), 128.04(11), $131.70\left(2^{\prime}, 6^{\prime}-\mathrm{Ph}\right), 135.90(9), 144.04(11 \mathrm{~b})$, 148.79 (3), 150.66 (6), 154.63 (7a), 160.20 (2), 162.55 (4'-Ph), 170.08 (COOH); LC-MS, $m / z=395$ [M+1], 397 [M+3]; Anal. Calcd for $\mathrm{C}_{19} \mathrm{H}_{14} \mathrm{~N}_{4} \mathrm{O}$ S: C, 57.86; H, 3.58; N, 14.21; S, 8.13; Found: C, 57.85; H, 3.54; N, 14.20; S, 8.12.

2-[(3-(4'-Ethoxyphenyl)-2-oxo-2 $H$ - $[1,2,4]$ triazino [2,3-c]quinazoline-6-yl)thio]acetic acids (3.8). Yield83.6\%. M.p. $-245-247^{\circ} \mathrm{C}$; LC-MS, m/z = 409 [M+1], 411 $[\mathrm{M}+3]$; Anal. calcd. for $\mathrm{C}_{20} \mathrm{H}_{16} \mathrm{~N}_{4} \mathrm{O}_{4} \mathrm{~S}$ : C, 58.81; H, 3.95; N, 13.72; S, 7.85; Found: C, 58.80; H, 3.95; N, 13.74; S, 7.87.

2-[(3-(4'-Fluorophenyl)-2-oxo-2H-[1,2,4]triazino [2,3-c] quinazoline-6-yl)thio]acetic acids (3.9). Yield76.2\%. M.p. $-230-232{ }^{\circ} \mathrm{C}$; ${ }^{1} \mathrm{H}$ NMR $\delta: 4.09$ (s, 2H, $-\mathrm{SCH}_{2}$ ), 9.46-6.62 (m, 8H, H-8, 9, 10, 11, 3-Ph H-2', 3', 5', 6' ), 12.83 (s, 1H, COOH); LC-MS, m/z = 383 [M+1], 385 [M+3]; Anal. calcd. for $\mathrm{C}_{18} \mathrm{H}_{11} \mathrm{FN}_{4} \mathrm{O}_{3} \mathrm{~S}$ : C, 56.54; H, 2.90; N, 14.65; S, 8.39; Found: C, 56.57; H, 2.90; N, 14.64; S, 8.38.

2-[(8-Methyl-3-phenyl)-2-oxo-2H-[1,2,4]triazino [2,3-c]quinazoline-6-yl)thio]acetic acids (3.10). Yield78.5\%. M.p. $-255-257^{\circ} \mathrm{C} ;{ }^{1} \mathrm{H}$ NMR $\delta: 2.65$ (s, 3H, $\left.\mathrm{CH}_{3}\right), 4.05$ (s, 2H, - $\mathrm{SCH}_{2}$ ), 7.66-7.41 (m, 4H, H-10, 3-Ph H-3', 4', 5'), 7.76 (d, $J=6.9 \mathrm{~Hz}, 1 \mathrm{H}, \mathrm{H}-9)$, 8.46-8.25 (m, 3H, H-11, 3-Ph H-2', 6'), 12.72 (s, 1H, COOH); LC-MS, m/z = 379 [M+1], $381[\mathrm{M}+3]$; Anal. calcd. for $\mathrm{C}_{19} \mathrm{H}_{14} \mathrm{~N}_{4} \mathrm{O}_{3} \mathrm{~S}: \mathrm{C}, 60.31$; H, 3.73; N, 14.81; S, 8.47; Found: C, 60.34; H, 3.73; N, 14.80; S, 8.45.

2-[(9-Fluoro-3-phenyl)-2-oxo-2H-[1,2,4]triazino [2,3-c]quinazoline-6-yl)thio]acetic acids (3.11). Yield- 
75.9\%. M.p. $-238-240{ }^{\circ} \mathrm{C} ;{ }^{1} \mathrm{HNMR} \delta: 4.08$ (s, $\left.2 \mathrm{H},-\mathrm{SCH}_{2}\right), 7.68-$ 7.39 (m, 5H, H-8, 10, 3-Ph H-3', 4', 5'), 8.32 (d, $J=7.0$ Hz, 2H, 3-Ph H-2', 6'), 8.60 (dd, $J=8.40,6.10 \mathrm{~Hz}, 1 \mathrm{H}, \mathrm{H}-11$ ), 12.94 (s, 1H, COOH); LC-MS, m/z = $383[\mathrm{M}+1], 385[\mathrm{M}+3]$; Anal. calcd. for $\mathrm{C}_{18} \mathrm{H}_{11} \mathrm{FN}_{4} \mathrm{O}_{3} \mathrm{~S}: \mathrm{C}, 56.54 ; \mathrm{H}, 2.90 ; \mathrm{N}, 14.65 ; \mathrm{S}$, 8.39; Found: C, 56.51; H, 2.90; N, 14.64; S, 8.41.

2-[(9-Fluoro-3-(4'-fluorophenyl)-2-oxo-2 H-[1,2,4]triazino[2,3-c]quinazoline-6-yl)thio]acetic acids (3.12). Yield $-50.6 \%$. M.p. $-247-249^{\circ} \mathrm{C}$; ${ }^{1} \mathrm{H}$ NMR $\delta: 4.08$ (s, 2H, $-\mathrm{SCH}_{2}$ ), 7.29 (t, 2H, 3-Ph H-3', 5'), 7.47-7.40 (m, 2H, H-8, 10), 8.42 (t, 2H, 3-Ph H-2', 6'), 8.48 (t, 1H, H-11); LC-MS, m/z = 401 $[\mathrm{M}+1], 403[\mathrm{M}+3]$; Anal. calcd. for $\mathrm{C}_{18} \mathrm{H}_{10} \mathrm{~F}_{2} \mathrm{~N}_{4} \mathrm{O}_{3} \mathrm{~S}$ : C, 54.00; H, 2.52; N, 13.99; S, 8.01; Found: C, 54.03; H, 2.52; N, 13.98; $\mathrm{S}, 8.03$.

2-[(10-Chloro-3-phenyl)-2-oxo-2H-[1,2,4]triazino [2,3-c]quinazoline-6-yl)thio]acetic acids (3.13). Yield64.6\%. M.p. - 258-260 ${ }^{\circ} \mathrm{C}$; ${ }^{1} \mathrm{H}$ NMR $\delta: 4.07$ (s, 2H, $-\mathrm{SCH}_{2}$ ), 7.65-7.39 (m, 3H, 3-Ph H-3', 4', 5'), 7.76 (d, $J=8.2 \mathrm{~Hz}, 1 \mathrm{H}$, H-8), 7.91 (d, J=8.2 Hz, 1H, H-9), 8.50-8.22 (m, 3H, H-11, 3-Ph H-2', 6'), 13.63 (s, 1H, COOH); LC-MS, m/z = 399 [M+1], $401[\mathrm{M}+3], 402[\mathrm{M}+4]$; Anal. calcd. for $\mathrm{C}_{18} \mathrm{H}_{11} \mathrm{ClN}_{4} \mathrm{O}_{3} \mathrm{~S}: \mathrm{C}$, 54.21; H, 2.78; N, 14.05; S, 8.04; Found: C, 54.24; H, 2.78; N, $14.03 ; \mathrm{S}, 8.02$.

2-[(10-Bromo-3-(4'-fluorophenyl)-2-oxo-2H-[1,2,4] triazino[2,3-c]quinazoline-6-yl)thio]acetic acids (3.14). Yield-70.5\%. M.p. $-257-259^{\circ} \mathrm{C}$; LC-MS, m/z $=461[\mathrm{M}]^{+}, 465$ $[\mathrm{M}+4]$; Anal. calcd. for $\mathrm{C}_{18} \mathrm{H}_{10} \mathrm{BrFN}_{4} \mathrm{O}_{3} \mathrm{~S}$ : C, 46.87; H, 2.19; N, 12.15; S, 6.95; Found: C, 46.88; H, 2.19; N, 12.16; S, 6.93.

1.3. General procedure for the synthesis of $N-R_{4}-[(8-$ $R_{1}-9-R_{2}-10-R_{3}-3-R-2-o x o-2 H-[1,2,4]$ triazino [2,3-c] quinazoline-6-yl)thio]acetamides (4.1-4.26). Add $0.93 \mathrm{~g}$ $(10 \mathrm{mmol})$ of chloracetamide or $10 \mathrm{mmol} \mathrm{N}-\mathrm{R}_{4}$-chloracetamides to the suspension of potassium of the proper 8- $\mathrm{R}_{1}-9-\mathrm{R}_{2}-10-\mathrm{R}_{3}-3-\mathrm{R}-2-$ oxo-2H-[1,2,4]triazino $[2,3-c]$ guinazoline-6-thiolates (2.1-2.18) $(10 \mathrm{mmol})$ in $20 \mathrm{ml}$ of propanol-2 and reflux for 1-1.5 hours. Cool the resulted mixture, filter the solid and dry. Crystallize the compounds obtained from dioxane-water mixture.

2-[(3-Methyl-2-oxo-2H-[1,2,4]triazino[2,3-c]quinazolin-6-yl)thio] acetamide (4.1). Yield-87.3\%. M.p. - 289$291^{\circ} \mathrm{C} ;{ }^{1} \mathrm{HNMR} \delta: 2.45$ (s, 3H, $\left.\mathrm{CH}_{3}\right), 3.93$ (s, 2H, $\left.-\mathrm{SCH}_{2}\right), 7.08$ $\left(\mathrm{s}, 1 \mathrm{H}, \mathrm{NH}_{2}\right), 7.50\left(\mathrm{~s}, 1 \mathrm{H}, \mathrm{NH}_{2}\right), 7.62(\mathrm{t}, J=8.2 \mathrm{~Hz}, 1 \mathrm{H}, \mathrm{H}-10)$, 7.77 (d, $J=8.2 \mathrm{~Hz}, 1 \mathrm{H}, \mathrm{H}-8), 7.91$ (t, $J=7.3 \mathrm{~Hz}, 1 \mathrm{H}, \mathrm{H}-9)$, 8.52 (d, $J=7.5 \mathrm{~Hz}, 1 \mathrm{H}, \mathrm{H}-11)$; LC-MS, m/z = 302 [M+1], $304[\mathrm{M}+3]$; Anal. calcd. for $\mathrm{C}_{13} \mathrm{H}_{11} \mathrm{~N}_{5} \mathrm{O}_{2} \mathrm{~S}$ : C, $51.82 ; \mathrm{H}, 3.68 ; \mathrm{N}$, 23.24; S, 10.64; Found: C, 51.82; H, 3.69; N, 23.24; S, 10.63.

2-[(3-Phenyl-2-oxo-2H-[1,2,4]triazino[2,3-c] quinazolin-6-yl)thio] acetamide (4.2). Yield - 82.3\%. M.p. - 285$288^{\circ} \mathrm{C}$; ${ }^{1} \mathrm{H}$ NMR $\delta: 3.98$ (s, 2H, $\left.-\mathrm{SCH}_{2}\right), 7.12$ (s, $\left.1 \mathrm{H}, \mathrm{NH}_{2}\right)$, 7.61-7.43 (m, 4H, 3-Ph 3,5, $\left.\mathrm{NH}_{2}\right), 7.66$ (t, $J=7.6 \mathrm{~Hz}, 1 \mathrm{H}$, $\mathrm{H}-10), 7.80$ (d, $J=7.7 \mathrm{~Hz}, 1 \mathrm{H}, \mathrm{H}-8), 7.94$ (t, $J=7.1 \mathrm{~Hz}, 1 \mathrm{H}$, H-9), 8.36 (d, $J=7.2 \mathrm{~Hz}, 2 \mathrm{H}, 3-\mathrm{Ph} \mathrm{H}-2,6), 8.56$ (d, $J=7.6 \mathrm{~Hz}$, $1 \mathrm{H}, \mathrm{H}-11)$; LC-MS, m/z = $366[\mathrm{M}+1]$; Anal. calcd. for $\mathrm{C}_{18} \mathrm{H}_{13} \mathrm{~N}_{5} \mathrm{O}_{2} \mathrm{~S}$ : C, 59.49; H, 3.61; N, 19.27; S, 8.82; Found: 59.48; $\mathrm{H}, 3.61 ; \mathrm{N}, 19.27 ; \mathrm{S}, 8.83$.

2-[(3-(4-Methylphenyl)-2-oxo-2H-[1,2,4] triazino [2,3-c]quinazolin-6-yl)thio]acetamide (4.3). Yield-69.3\%. M.p. $-264-267^{\circ} \mathrm{C}$; IR $\left(\mathrm{cm}^{-1}\right): 3434,3314,1682,1664,1589$, 1561, 1543, 1496, 1469, 1400, 1367, 1341, 1311, 1272, 1240, $1190,1161,1135,1108,1075,1021,991,940,885,833,784$,
772, 707, 686, 643, 629; ${ }^{1} \mathrm{H}$ NMR $\delta: 2.39$ (s, 3H, $\left.\mathrm{CH}_{3}\right), 4.00$ (s, $\left.2 \mathrm{H},-\mathrm{SCH}_{2}\right), 7.30\left(\mathrm{~s}, 1 \mathrm{H},-\mathrm{C}(\mathrm{O}) \mathrm{NH}_{2}\right), 7.39\left(\mathrm{~d}, 2 \mathrm{H}, J=8.2, \mathrm{H}-3^{\prime}\right.$, $\left.5^{\prime} \mathrm{Ph}\right), 7.78-7.64$ (m, 3H, H-8, 10, -C(O)NH$), 7.96$ (t, $1 \mathrm{H}, J=$ 7.9, H-9), 8.22 (d, 2H, $\left.J=8.2, \mathrm{H}-2^{\prime}, 6^{\prime} \mathrm{Ph}\right), 8.46(\mathrm{~d}, 1 \mathrm{H}, J=7.9$, H-11); LC-MS, m/z = 378 [M+1], 380 [M+3]; Anal. calcd. for $\mathrm{C}_{19} \mathrm{H}_{15} \mathrm{~N}_{5} \mathrm{O}_{2} \mathrm{~S}: \mathrm{C}, 60.47$; H, 4.01; N, 18.56, S, 8.50; Found: C, 59.47; H, 4.03; N, 18.55; S, 8.52.

2-[(3-(3,4-Dimethylphenyl)-2-oxo-2H-[1,2,4]triazino[2,3-c]quinazolin-6-yl)thio] acetamide (4.4). Yield -94.05\%. M.p. $-272-275^{\circ} \mathrm{C}$; ${ }^{1} \mathrm{H}$ NMR $\delta: 2.33$ (s, 3H, 3-CH ${ }_{3}$, 2.37 (s, 3H, 4-CH $), 3.95$ (s, 2H, $\left.-\mathrm{SCH}_{2}\right), 7.07$ (s, 1H, NH 7.51 (s, 1H, NH$\left.)_{2}\right), 7.28$ (d, J=7.9 Hz, 1H, 3-Ph H-5'), 7.63 (t, $J=8.2 \mathrm{~Hz}, 1 \mathrm{H}, \mathrm{H}-10), 7.76$ (d, $J=8.2 \mathrm{~Hz}, 1 \mathrm{H}, \mathrm{H}-8), 7.90$ (t, $J$ $=7.3 \mathrm{~Hz}, 1 \mathrm{H}, \mathrm{H}-9), 8.03$ (d, $\left.J=7.8 \mathrm{~Hz}, 1 \mathrm{H}, 3-\mathrm{Ph} \mathrm{H}-6^{\prime}\right), 8.12$ (s, 1H, 3-Ph H-2'), 8.53 (d, $J=7.5$ Hz, 1H, H-11); LC-MS, $\mathrm{m} / \mathrm{z}=392[\mathrm{M}+1], 394[\mathrm{M}+4]$; Anal. calcd. for $\mathrm{C}_{20} \mathrm{H}_{17} \mathrm{~N}_{5} \mathrm{O}_{2} \mathrm{~S}: \mathrm{C}$, 61.37; H, 4.38; N, 17.89; S, 8.19; Found: C, 61.36; H, 4.38; N, $17.89 ; \mathrm{S}, 8.20$.

2-[(3-(4'-Ethylphenyl)-2-oxo-2H-[1,2,4]triazino [2,3-c] quinazolin-6-yl)thio] acetamide (4.5). Yield-86.4\%. M.p. $-233-235^{\circ} \mathrm{C} ;{ }^{1} \mathrm{H}$ NMR $\delta: 1.30$ (t, J = 7.4 Hz, $3 \mathrm{H} \mathrm{CH}_{3}$ ), $2.75\left(\mathrm{dd}, \mathrm{J}=14.8,7.2 \mathrm{~Hz}, 2 \mathrm{H}, \mathrm{CH}_{2} \mathrm{CH}_{3}\right), 3.97$ (s, 2H, $-\mathrm{SCH}_{2}$ ), $7.13\left(\mathrm{~s}, 1 \mathrm{H}, \mathrm{NH}_{2}\right), 7.35$ (d, J = 8.0 Hz, 2H, 3-Ph H-3, 5), 7.54 (s, $\left.1 \mathrm{H}, \mathrm{NH}_{2}\right), 7.63(\mathrm{t}, \mathrm{J}=7.5 \mathrm{~Hz}, 1 \mathrm{H}, \mathrm{H}-10), 7.78(\mathrm{~d}, \mathrm{~J}=8.1 \mathrm{~Hz}, 1 \mathrm{H}$, $\mathrm{H}-8), 7.91$ (t, J=7.3 Hz, 1H, H-9), 8.29 (d, J=8.0 Hz, 2H, 3-Ph $\mathrm{H}-2,6), 8.53$ (d, J = 7.8 Hz, 1H, H-11); LC-MS, m/z = 392 [M+1], 394 [M+3]; Anal. calcd. for $\mathrm{C}_{20} \mathrm{H}_{17} \mathrm{~N}_{5} \mathrm{O}_{2} \mathrm{~S}$ : C, 61.37; H, 4.38; N, 17.89; S, 8.19; Found: C, 61.39; H, 4.38; N, 17.88; S, 8.18.

2-[(3-(4'-(tert-Butyl)phenyl)-2-oxo-2H-[1,2,4]triazino[2,3-c] quinazolin-6-yl)thio]acetamide (4.6). Yield - 79.5\%. M.p. $-265-268^{\circ} \mathrm{C}$; ${ }^{1} \mathrm{H}$ NMR $\delta: 1.39$ (s, 9H, $\left.\mathrm{C}\left(\mathrm{CH}_{3}\right)_{3}\right), 3.97$ (s, $\left.2 \mathrm{H},-\mathrm{SCH}_{2}\right), 7.13\left(\mathrm{~s}, 1 \mathrm{H}, \mathrm{NH}_{2}\right), 7.54(\mathrm{~d}, J=7.9 \mathrm{~Hz}, 3 \mathrm{H}, 3-\mathrm{Ph}$ $\left.\mathrm{H}-3,5, \mathrm{NH}_{2}\right), 7.62$ (t, $\left.J=7.4 \mathrm{~Hz}, 1 \mathrm{H}, \mathrm{H}-10\right), 7.77$ (d, $J=8.0 \mathrm{~Hz}$, $1 \mathrm{H}, \mathrm{H}-8), 7.90$ (t, $J=7.3 \mathrm{~Hz}, 1 \mathrm{H}, \mathrm{H}-9), 8.30$ (d, $J=8.2 \mathrm{~Hz}, 2 \mathrm{H}, 3-\mathrm{Ph}$ $\mathrm{H}-2,6), 8.53$ (d, $J=7.8 \mathrm{~Hz}, 1 \mathrm{H}, \mathrm{H}-11)$; LC-MS, m/z = 420 [M+1], $422[\mathrm{M}+3]$; Anal. calcd. for $\mathrm{C}_{22} \mathrm{H}_{21} \mathrm{~N}_{5} \mathrm{O}_{2} \mathrm{~S}$ : C, 62.99; H, 5.05; N, 16.69; S, 7.64; Found: C, 62.99; H, 5.06; N, 16.69; S, 7.63.

2-[(3-(4'-Methoxyphenyl)-2-oxo-2H-[1,2,4]triazino[2,3-c]quinazolin-6-yl)thio]acetamide (4.7). Yield - 88.9\%. M.p. $-253-255^{\circ} \mathrm{C} ;{ }^{1} \mathrm{H}$ NMR $\delta: 3.90$ (s, 3H, $\left.\mathrm{CH}_{3}\right), 3.97$ (s, 2H, $\left.-\mathrm{SCH}_{2}\right), 7.07$ (d, $\left.J=8.2 \mathrm{~Hz}, 2 \mathrm{H}, 3-\mathrm{PhH}-3^{\prime}, 5^{\prime}\right), 7.13\left(\mathrm{~s}, 1 \mathrm{HNH}_{2}\right)$, $7.55\left(\mathrm{~s}, 1 \mathrm{H}, \mathrm{NH}_{2}\right), 7.64$ (t, $\left.J=7.5 \mathrm{~Hz}, 1 \mathrm{H}, \mathrm{H}-10\right), 7.79$ (d, $J=$ $7.6 \mathrm{~Hz}, 1 \mathrm{H}, \mathrm{H}-8), 7.92(\mathrm{t}, J=7.5 \mathrm{~Hz}, 1 \mathrm{H}, \mathrm{H}-9), 8.42(\mathrm{~d}, J=8.2 \mathrm{~Hz}$, 2H, 3-Ph H-2',6'), 8.54 (d, $J=7.1 \mathrm{~Hz}, 1 \mathrm{H}, \mathrm{H}-11)$; LC-MS, $\mathrm{m} / \mathrm{z}=394[\mathrm{M}+1], 396[\mathrm{M}+3]$; Anal. calcd. for $\mathrm{C}_{19} \mathrm{H}_{15} \mathrm{~N}_{5} \mathrm{O}_{3} \mathrm{~S}$ : C, 58.01; H, 3.84; N, 17.80; S, 8.15; Found: C, 58.00; H, 3.84; N, 17.80; S, 8.16.

2-[(3-(4'-Ethoxyphenyl)-2-oxo- $2 H$ - $[1,2,4]$ triazino [2,3-c] quinazolin-6-yl)thio]acetamide (4.8). Yield-91.6\%. M.p. $-288-291{ }^{\circ} \mathrm{C} ;{ }^{1} \mathrm{H}$ NMR $\delta: 1.44$ (t, 3H, $\left.\mathrm{CH}_{3}\right), 3.96$ (s, 2H, $\left.-\mathrm{SCH}_{2}\right), 4.14\left(\mathrm{q}, \mathrm{J}=6.3 \mathrm{~Hz}, 2 \mathrm{H}, \mathrm{OCH}_{2} \mathrm{CH}_{3}\right), 6.99(\mathrm{~d}, \mathrm{~J}=8.0 \mathrm{~Hz}$, 2H, 3-Ph H-3', 5'), 7.14 (s, 1H, $\mathrm{NH}_{2}$ ), 7.53 (s, 1H, $\left.\mathrm{NH}_{2}\right), 7.64$ (t, J = 7.6 Hz, 1H, H-10), 7.80 (d, J = 7.6 Hz, 1H, H-8), 7.93 (t, $\mathrm{J}=7.6 \mathrm{~Hz}, 1 \mathrm{H}, \mathrm{H}-9), 8.42$ (d, J = 8.0 Hz, 2H, 3-Ph H-2', 6'), $8.53(\mathrm{~d}, \mathrm{~J}=7.6 \mathrm{~Hz}, 1 \mathrm{H}, \mathrm{H}-11)$; Anal. calcd. for $\mathrm{C}_{20} \mathrm{H}_{17} \mathrm{~N}_{5} \mathrm{O}_{3} \mathrm{~S}$ : C, 58.96; H, 4.21; N, 17.19; S, 7.87; Found: C, 58.96; H, 4.22; N, 17.19; S, 7.86.

$\mathrm{N}$-(3-Fluorophenyl)-2-[(3-(4'-methoxyphenyl)-2-oxo$2 H$-[1,2,4]triazino[2,3-c] quinazolin-6-yl)thio]acetamide (4.9). Yield-87.8\%. M.p. $-274-276^{\circ} \mathrm{C} ;{ }^{1} \mathrm{H}$ NMR $\delta: 3.91$ 
$\left(\mathrm{s}, 3 \mathrm{H},-\mathrm{OCH}_{3}\right), 4.22\left(\mathrm{~s}, 2 \mathrm{H},-\mathrm{SCH}_{2}\right), 6.78(\mathrm{dd}, J=10.7,8.3 \mathrm{~Hz}$, 1H, 6-Ph H-4), 7.08 (d, $J=8.8$ Hz, 2H, 3-Ph H-3',5'), 7.447.22 (m, 2H, 6-Ph H-2, 6), 7.69 - 7.54 (m, 2H, H-10, 6-Ph H-5), 7.74 (d, $J=8.8 \mathrm{~Hz}, 1 \mathrm{H}, \mathrm{H}-8), 7.91$ (t, $J=7.8,1 \mathrm{H}, \mathrm{H}-9)$, 8.44 (d, $\left.J=8.2 \mathrm{~Hz}, 2 \mathrm{H}, 3-\mathrm{Ph} \mathrm{H}-2^{\prime}, 6^{\prime}\right), 8.54$ (d, $J=7.8 \mathrm{~Hz}$, 1H, H-11), 10.53 (s, 1H, NH); LC-MS, m/z = 488 [M+1], 490 $[\mathrm{M}+3]$; Anal. calcd. for $\mathrm{C}_{25} \mathrm{H}_{18} \mathrm{FN}_{5} \mathrm{O}_{3} \mathrm{~S}: \mathrm{C}, 61.59 ; \mathrm{H}, 3.72 ; \mathrm{N}$, 14.37; S, 6.58; Found: C, 61.61; H, 3.72; N, 14.36; S, 6.57.

2-[(3-(4'-Fluorophenyl)-2-oxo-2H-[1,2,4]triazino [2,3-c]quinazolin-6-yl)thio]acetamide (4.10). Yield - 81.1\%. M.p. $-284-287^{\circ} \mathrm{C} ;{ }^{1} \mathrm{HNMR} \delta: 3.95$ (s, $\left.2 \mathrm{H},-\mathrm{SCH}_{2}\right), 7.12(\mathrm{~s}, 1 \mathrm{H}$, $\left.\mathrm{NH}_{2}\right), 7.29$ (t, $\left.J=8.5 \mathrm{~Hz}, 2 \mathrm{H}, 3-\mathrm{PhH}-3^{\prime}, 5^{\prime}\right), 7.55\left(\mathrm{~s}, 1 \mathrm{H}, \mathrm{NH}_{2}\right), 7.71$ (t, $J=7.4 \mathrm{~Hz}, 1 \mathrm{H}, \mathrm{H}-10), 7.89$ (d, $J=8.0 \mathrm{~Hz}, 1 \mathrm{H}, \mathrm{H}-8), 7.99$ (t, $J=7.3 \mathrm{~Hz}, 1 \mathrm{H}, \mathrm{H}-9), 8.43$ (dd, $J=7.5,5.9 \mathrm{~Hz}, 2 \mathrm{H}, 3-\mathrm{Ph} \mathrm{H}-2^{\prime}$, $\left.6^{\prime}\right), 8.59$ (d, $\left.J=7.9 \mathrm{~Hz}, 1 \mathrm{H}, \mathrm{H}-11\right)$; LC-MS, m/z = 382 [M+1], $384[\mathrm{M}+3]$; Anal. calcd. for $\mathrm{C}_{18} \mathrm{H}_{12} \mathrm{FN}_{5} \mathrm{O}_{2} \mathrm{~S}$ : C, 56.69; H, 3.17; 18.36; S, 8.41; Found: C, 56.68; H, 3.17; 18.376; S, 8.41.

2-[(8-Methyl-3-phenyl-2-oxo-2H-[1,2,4]triazino [2,3-c]quinazolin-6-yl)thio]acetamide (4.11). Yield-86.2\%. M.p. $-282-284^{\circ} \mathrm{C} ;{ }^{1} \mathrm{H}$ NMR $\delta: 2.75$ (s, 3H, $\left.\mathrm{CH}_{3}\right), 3.98$ (s, 2H, $\left.-\mathrm{SCH}_{2}\right), 7.15\left(\mathrm{~s}, 1 \mathrm{H}, \mathrm{NH}_{2}\right), 7.62-7.43$ (m, 5H, H-10, 3-Ph H-3', $\left.4^{\prime}, 5^{\prime}, \mathrm{NH}_{2}\right), 7.82$ (d, $\left.J=5.6 \mathrm{~Hz}, 1 \mathrm{H}, \mathrm{H}-9\right), 8.30$ (d, $J=8.8 \mathrm{~Hz}$, 2H, 3-Ph H-2', 6'), 8.43 (d, $J=7.8 \mathrm{~Hz}, 1 \mathrm{H}, \mathrm{H}-11$ ); Anal. calcd. for $\mathrm{C}_{19} \mathrm{H}_{15} \mathrm{~N}_{5} \mathrm{O}_{2} \mathrm{~S}$ : C, 60.46; H, 4.01; N, 18.56; S, 8.50; Found: C, 60.45; H, 4.01; N, 18.56; S, 8.51.

2-[(8-Bromo-3-(4-fluorophenyl)-2-oxo-2H-[1,2,4]triazino[2,3-c] quinazolin-6-yl)thio]acetamide (4.12). Yield $-79.5 \%$. M.p. $-270-273^{\circ} \mathrm{C}$; ${ }^{1} \mathrm{H}$ NMR $\delta: 3.94$ (s, 2H, - $\left.\mathrm{SCH}_{2}\right)$, $6.60(\mathrm{t}, J=7.6 \mathrm{~Hz}, 1 \mathrm{H}, \mathrm{H}-10), 7.15\left(\mathrm{~s}, 1 \mathrm{H}, \mathrm{NH}_{2}\right), 7.21(\mathrm{t}, J$ $\left.=7.8 \mathrm{~Hz}, 2 \mathrm{H}, 3-\mathrm{Ph} \mathrm{H}-3^{\prime}, 5^{\prime}\right), 7.54\left(\mathrm{~s}, 1 \mathrm{H}, \mathrm{NH}_{2}\right), 7.66(\mathrm{~d}, J=$ $7.4 \mathrm{~Hz}, 1 \mathrm{H}, \mathrm{H}-9), 7.75$ (d, J=7.7 Hz, 1H, H-11), 8.33 (t, 2H, J = $\left.5.3 \mathrm{~Hz}, 3-\mathrm{Ph} \mathrm{H}-2^{\prime}, 6^{\prime}\right)$; LC-MS, m/z = 460 [M]; Anal. calcd. for $\mathrm{C}_{18} \mathrm{H}_{11} \mathrm{BrFN}_{5} \mathrm{O}_{2} \mathrm{~S}: \mathrm{C}, 46.97 ; \mathrm{H}, 2.41 ; \mathrm{N}, 15.22 ; \mathrm{S}, 6.97$; Found: C, 46.96; H, 2.41; N, 15.22; S, 6.98 .

2-[(9-Fluoro-3-phenyl-2-oxo-2 $H$-[1,2,4]triazino [2,3-c] quinazolin-6-yl)thio]acetamide (4.13). Yield - 91.2\%. M.p. $-286-289^{\circ} \mathrm{C}$; ${ }^{1} \mathrm{HNMR} \delta: 3.95\left(\mathrm{~s}, 2 \mathrm{H},-\mathrm{SCH}_{2}\right), 7.13(\mathrm{~s}, 1 \mathrm{H}$, $\mathrm{NH}_{2}$ ), 7.47-7.36 (t, 1H, H-10), 7.54 (m, 5H, H-8, 3-Ph H-3', 4', $\left.5^{\prime}, \mathrm{NH}_{2}\right), 8.33$ (d, J = 5.7 Hz, 2H, 3-Ph H-2',6'), 8.59 (dd, J = 6.2, 4.6 Hz, 1H, H-11); LC-MS, m/z = 382 [M+1], $384[\mathrm{M}+3]$; Anal. calcd. for $\mathrm{C}_{18} \mathrm{H}_{12} \mathrm{FN}_{5} \mathrm{O}_{2} \mathrm{~S}$ : C, 56.69; H, 3.17; N, 18.36; S, 8.41; Found: C, 56.69; H, 3.16; N, 18.36; S, 8.42.

2-[(9-Fluoro-3-(4'-methoxyphenyl)-2-oxo-2H$[1,2,4]$ triazino[2,3-c]quinazolin-6-yl)thio]acetamide (4.14). Yield - 89.1\%. M.p. - 251-254 ${ }^{\circ} \mathrm{C} ;{ }^{1} \mathrm{H}$ NMR $\delta: 3.89$ $\left(\mathrm{s}, 3 \mathrm{H},-\mathrm{OCH}_{3}\right), 3.95\left(\mathrm{~s}, 2 \mathrm{H},-\mathrm{SCH}_{2}\right), 7.05(\mathrm{~d}, J=8.6 \mathrm{~Hz}, 2 \mathrm{H}$, 3-Ph H-3', 5'), 7.14 (s, 1H, NH $), 7.43$ (dd, $J=8.6,6.5 \mathrm{~Hz}, 1 \mathrm{H}$, H-10), 7.51 (d, $J=8.7 \mathrm{~Hz}, 1 \mathrm{H}, \mathrm{H}-8), 7.56$ (s, 1H, NH$), 8.40$ (d, $J=8.7 \mathrm{~Hz}, 2 \mathrm{H}, 3-\mathrm{Ph} \mathrm{H}-2,6), 8.59$ (dd, $J=8.0,5.4 \mathrm{~Hz}, 1 \mathrm{H}$, H-11); LC-MS, m/z = 412 [M+1], 414 [M+3]; Anal. calcd. for $\mathrm{C}_{19} \mathrm{H}_{14} \mathrm{FN}_{5} \mathrm{O}_{3} \mathrm{~S}: \mathrm{C}, 55.47 ; \mathrm{H}, 3.43 ; \mathrm{N}, 17.02 ; \mathrm{S}, 7.79$; Found: C, $55.43 ; \mathrm{H}, 3.46 ; \mathrm{N}, 17.01 ; \mathrm{S}, 7.80$.

$N$-(4-Bromophenyl)-2-[(9-fluoro-3-(4-methoxyphenyl)-2-oxo-2 $H$-[1,2,4]triazino[2,3-c]quinazolin-6-yl)thio] acetamide (4.15). Yield - 92.6\%. M.p. $-304-306^{\circ} \mathrm{C}$; ${ }^{1} \mathrm{H}$ NMR $\delta: 3.90\left(\mathrm{~s}, 3 \mathrm{H},-\mathrm{OCH}_{3}\right), 4.20\left(\mathrm{~s}, 2 \mathrm{H},-\mathrm{SCH}_{2}\right), 7.08(\mathrm{~d}, J$ $\left.=8.7 \mathrm{~Hz}, 2 \mathrm{H}, 3-\mathrm{Ph} \mathrm{H}-3^{\prime}, 5^{\prime}\right), 7.51-7.32(\mathrm{~m}, 4 \mathrm{H}, \mathrm{H}-8,10,6-\mathrm{Ph}$ H-2', 6'), 7.62 (d, $\left.J=8.5 \mathrm{~Hz}, 2 \mathrm{H}, 6-\mathrm{Ph} \mathrm{H}-3^{\prime}, 5^{\prime}\right), 8.41$ (d, $J=$ 8.7 Hz, 2H, 3-PhH-2', 6'), 8.57 (dd, $J=8.4,6.3 \mathrm{~Hz}, 1 \mathrm{H}, \mathrm{H}-11$ ), 10.46 (s, $1 \mathrm{H}, \mathrm{NH})$; Anal. calcd. for $\mathrm{C}_{25} \mathrm{H}_{17} \mathrm{BrFN}_{5} \mathrm{O}_{3} \mathrm{~S}: \mathrm{C}, 53.01$;
H, 3.03; Br, 14.11; F, 3.35; N, 12.36; S, 5.66; Found: C, 53.04; H, 3.03; Br, 14.11; F, 3.35; N, 12.34; S, 5.65.

2-[(9-Fluoro-3-(4-fluorophenyl)-2-oxo-2H-[1,2,4]triazino[2,3-c] quinazolin-6-yl)thio]acetamide (4.16). Yield $-71.8 \%$. M.p. $-274-276^{\circ} \mathrm{C}$; ${ }^{1} \mathrm{H}$ NMR $\delta: 3.96$ (s, 2H, $\left.-\mathrm{SCH}_{2}\right)$; 7.17 (s, 1H, NH $\left.\mathrm{N}_{2}\right), 7.28$ (t, $J=8.4$ Hz, 2H, 3-Ph H-3' '5') 7.53 (t, $J=7.5 \mathrm{~Hz}, 1 \mathrm{H}, \mathrm{H}-10), 7.55\left(\mathrm{~s}, 1 \mathrm{H}, \mathrm{NH}_{2}\right), 7.56(\mathrm{~d}, J=9.2 \mathrm{~Hz}$, 1H, H-8), 8.39 (t, $J=5.7$ Hz, 2H, 3-Ph H-2',6'), 8.65 (dd, $J=$ 8.3, 6.0 Hz, 1H, H-11); LC-MS, m/z = 400 [M+1], 402 [M+3]; Anal. calcd. for $\mathrm{C}_{18} \mathrm{H}_{11} \mathrm{~F}_{2} \mathrm{~N}_{5} \mathrm{O} \mathrm{O}_{2} \mathrm{~S}: \mathrm{C}, 54.13 ; \mathrm{H}, 2.78 ; \mathrm{N}, 17.54 ; \mathrm{S}$, 8.03; Found: C, 54.12; H, 2.78; N, 17.55; S, 8.03.

2-[(9-Bromo-3-(4-fluorophenyl)-2-oxo-2H-[1,2,4]triazino[2,3-c] quinazolin-6-yl)thio] acetamide (4.17). Yield -84.62\%. M.p. $-282-285^{\circ} \mathrm{C} ;{ }^{1} \mathrm{H}$ NMR $\delta: 3.95$ (s, 2H, $-\mathrm{SCH}_{2}$ ); 7.12 (s, 1H, NH $), 7.30$ (t, $J=8.4$ Hz, 2H, 3-Ph H-3,5), 7.75 $(\mathrm{d}, J=8.1 \mathrm{~Hz}, 1 \mathrm{H}, \mathrm{H}-10), 7.55\left(\mathrm{~s}, 1 \mathrm{H}, \mathrm{NH}_{2}\right), 8.00(\mathrm{~s}, 1 \mathrm{H}, \mathrm{H}-8)$, 8.55-8.32 (m, 3H, H-11, 3-Ph H-2,6); LC-MS, m/z = 461 $[\mathrm{M}+1]$; Anal. calcd. for $\mathrm{C}_{18} \mathrm{H}_{11} \mathrm{BrFN}_{5} \mathrm{O}_{2} \mathrm{~S}$ : C, 46.97; $\mathrm{H}, 2.41$; N, 15.22; S, 6.97; Found: C, 46.97; H, 2.42; N, 15.22; S, 6.96.

N-(4-(Trifluoromethyl)benzyl)-2-[(9-bromo-3-(4-fluorophenyl)-2-oxo-2H-[1,2,4]triazino[2,3-c] quinazolin-6yl)thio] acetamide (4.18). Yield-81.9\%. M.p. $-273-276^{\circ} \mathrm{C}$; ${ }^{1} \mathrm{HNMR} \delta: 4.04\left(\mathrm{~s}, 2 \mathrm{H},-\mathrm{SCH}_{2}\right), 4.44\left(\mathrm{~d}, J=4.6 \mathrm{~Hz}, 2 \mathrm{H},-\mathrm{CH}_{2}\right)$, 7.33 (t, $J=7.7$ Hz, 2H, 3-Ph H-3', 5'), 7.57-7.41 (m, 4H, 6-Ph H-2', 3', 5', 6'), 7.79 (d, J=8.4 Hz, 1H, H-10), 7.90 (s, 1H, H-8), 8.51-8.33 (m, 3H, H-11, 3-Ph H-2', 6'), 8.80 (t, $J=5.9$ Hz, $1 \mathrm{H}, \mathrm{NHCO}$ ); Anal. calcd. for $\mathrm{C}_{26} \mathrm{H}_{16} \mathrm{BrF}_{4} \mathrm{~N}_{5} \mathrm{O} 2 \mathrm{~S}: \mathrm{C}, 50.50 ; \mathrm{H}$, 2.61; Br, 12.92; F, 12.29; N, 11.32; S, 5.19; Found: C, 50.53; H, 2.61; Br, 12.92; F, 12.29; N, 11.31; S, 5.17.

2-[(10-Chloro-3-phenyl-2-oxo-2H-[1,2,4]triazino [2,3-c] quinazolin-6-yl)thio] acetamide (4.19). Yield 65.9\%. M.p.-273-276 ${ }^{\circ} \mathrm{C}$; ${ }^{1} \mathrm{HNMR} \delta: 3.97$ (s, 2H, $\left.-\mathrm{SCH}_{2}\right), 7.13$ $\left(\mathrm{s}, 1 \mathrm{H}, \mathrm{NH}_{2}\right), 7.66-7.39$ (m, 3H, 3-PhH-3', 5', $\left.\mathrm{NH}_{2}\right), 7.81(\mathrm{~d}, J=$ $8.7 \mathrm{~Hz}, 1 \mathrm{H}, \mathrm{H}-8), 7.89$ (d, $J=8.7 \mathrm{~Hz}, 1 \mathrm{H}, \mathrm{H}-9), 8.36$ (d, $J=$ $7.1 \mathrm{~Hz}, 2 \mathrm{H}, 3-\mathrm{Ph} \mathrm{H}-2^{\prime}, 6^{\prime}$ ), 8.47 (s, 1H, H-11); LC-MS, m/z = 398 $[\mathrm{M}+1], 400[\mathrm{M}+3], 401[\mathrm{M}+4]$; Anal. calcd. for $\mathrm{C}_{18} \mathrm{H}_{12} \mathrm{CIN}_{5} \mathrm{O}_{2} \mathrm{~S}$ : C, 54.34; H, 3.04; N, 17.60; S, 8.06; Found: C, 54.36; H, 3.04; N, 17.59; S, 8.05.

$N$-(4-Methoxybenzyl)-2-[(10-chloro-2-oxo-3-phenyl$2 H$-[1,2,4] triazino[2,3-c] quinazolin-6-yl)thio] acetamide (4.20). Yield-81.4\%. M.p. $-242-244^{\circ} \mathrm{C} ;{ }^{1} \mathrm{HNMR} \delta: 3.74$ $\left(\mathrm{s}, 3 \mathrm{H}, \mathrm{OCH}_{3}\right), 4.01\left(\mathrm{~s}, 2 \mathrm{H},-\mathrm{SCH}_{2}\right), 4.27(\mathrm{~d}, J=5.5 \mathrm{~Hz}, 2 \mathrm{H}$, $\left.-\mathrm{CH}_{2}\right), 6.74\left(\mathrm{~d}, J=8.2 \mathrm{~Hz}, 2 \mathrm{H}, 6-\mathrm{Ph} \mathrm{H}-3^{\prime}, 5^{\prime}\right), 7.17$ (d, $J=8.1$ Hz, 2H, 6-Ph H-2', 6'), 7.66-7.46 (m, 4H, H-8, 3-Ph H-3', 4', $\left.5^{\prime}\right), 7.86(\mathrm{~d}, J=8.6 \mathrm{~Hz}, 1 \mathrm{H}, \mathrm{H}-9), 8.35$ (d, $J=7.3 \mathrm{~Hz}, 2 \mathrm{H}$, 3-Ph H-2', 6'), 8.45 (s, 1H, H-11), 8.60 (t, $J=5.5$ Hz, 1H, $\mathrm{NH})$; LC-MS, m/z = $519[\mathrm{M}+1], 522[\mathrm{M}+4]$; Anal. calcd. for $\mathrm{C}_{26} \mathrm{H}_{20} \mathrm{CIN}_{5} \mathrm{O}_{3} \mathrm{~S}: \mathrm{C}, 60.29 ; \mathrm{H}, 3.89 ; \mathrm{Cl}, 6.84 ; \mathrm{N}, 13.52 ; \mathrm{S}, 6.19$; Found: C, 60.32; H, 3.89; Cl, 6.84; N, 13.50; S, 6.22.

N-(4-(Trifluoromethyl)benzyl)-2-[(10-chloro-3(4-methoxyphenyl)-2-oxo-2H-[1,2,4] triazino[2,3-c] quinazolin-6-yl)thio]acetamide (4.21). Yield - 93.5\%. M.p. $-279-282^{\circ} \mathrm{C} ;{ }^{1} \mathrm{H}$ NMR $\delta: 3.91$ (s, 3H, $\left.-\mathrm{OCH}_{3}\right), 4.05$ (s, $\left.2 \mathrm{H},-\mathrm{SCH}_{2}\right), 4.43\left(\mathrm{~d}, J=3.8 \mathrm{~Hz}, 2 \mathrm{H},-\mathrm{CH}_{2}\right), 7.08(\mathrm{~d}, J=7.6 \mathrm{~Hz}$, 2H, 3-Ph H-3', 5'), 7.54-7.40 (m, 5H, 6-Ph H-2',3',4', 5', 6'), 7.64 (d, $J=7.8 \mathrm{~Hz}, 1 \mathrm{H}, \mathrm{H}-8), 7.84$ (d, $J=7.9 \mathrm{~Hz}, 1 \mathrm{H}, \mathrm{H}-9)$, 8.51-8.33 (m, 3H, H-11, 3-Ph H-2', 6'), 8.82 (t, J=3.8 Hz, 1H, $\mathrm{NH}$ ); Anal. calcd. for $\mathrm{C}_{27} \mathrm{H}_{19} \mathrm{ClF}_{3} \mathrm{~N}_{5} \mathrm{O}_{3} \mathrm{~S}: \mathrm{C}, 55.34 ; \mathrm{H}, 3.27 ; \mathrm{Cl}$, 6.05; F, 9.73; N, 11.95; S, 5.47; Found: C, 55.36; H, 3.27; Cl, $6.05 ; \mathrm{F}, 9.73 ; \mathrm{N}, 11.94 ; \mathrm{S}, 5.46$. 
2-[(10-Bromo-3-phenyl-2-oxo-2H-[1,2,4]triazino [2,3-c] quinazolin-6-yl)thio] acetamide (4.23). Yield - 64.0\%. M.p. $-250-253^{\circ} \mathrm{C}$; ${ }^{1} \mathrm{HNMR} \delta: 3.97$ (s, $\left.2 \mathrm{H},-\mathrm{SCH}_{2}\right), 7.13$ (s, $1 \mathrm{H}$, $\mathrm{NH}_{2}$ ), 7.65-7.46 (m, 4H, 3-Ph H-3', 4', 5', NH $), 7.74$ (d, $J=$ $7.9 \mathrm{~Hz}, 1 \mathrm{H}, \mathrm{H}-8), 8.02(\mathrm{~d}, J=7.8 \mathrm{~Hz}, 1 \mathrm{H}, \mathrm{H}-9), 8.36(\mathrm{~d}, J=6.9 \mathrm{~Hz}$, 2H, 3Ph H-2',6'), 8.63 (s, 1H, H-11); LC-MS, m/z = 444 [M+2], 446 [M+4]; Anal. calcd. for $\mathrm{C}_{18} \mathrm{H}_{12} \mathrm{BrN}_{5} \mathrm{O}_{2} \mathrm{~S}: \mathrm{C}, 48.88$; $\mathrm{H}, 2.73$; N, 15.83; S, 7.25; Found: C, 48.87; H, 2.74; N, 15.83; S, 7.25.

2-[(10-Bromo-3-(4-methylphenyl)-2-oxo-2 $H$-[1,2,4] triazino[2,3-c] quinazolin-6-yl)thio]acetamide (4.23). Yield $-79.5 \%$. M.p. $-275-278^{\circ} \mathrm{C} ;{ }^{1} \mathrm{H}$ NMR $\delta: 2.45$ (s, 3H, $-\mathrm{CH}_{3}$ ), 3.96 (s, 2H, $\left.-\mathrm{SCH}_{2}\right), 6.32$ (d, $\left.J=7.9 \mathrm{~Hz}, 2 \mathrm{H}, 3-\mathrm{Ph} \mathrm{H}-3{ }^{\prime}, 5^{\prime}\right)$, $7.13\left(\mathrm{~s}, 1 \mathrm{H}, \mathrm{NH}_{2}\right), 7.57\left(\mathrm{~s}, 1 \mathrm{H}, \mathrm{NH}_{2}\right), 7.81(\mathrm{~d}, J=8.6 \mathrm{~Hz}, 1 \mathrm{H}$, H-8), 8.07 (dd, $J=8.6,1.6 \mathrm{~Hz}, 1 \mathrm{H}, \mathrm{H}-9), 8.23$ (d, $J=8.0 \mathrm{~Hz}$, 2H, 3-Ph H-2', 6'), 8.64 (s, 1H, H-11); LC-MS, m/z = 457 $[\mathrm{M}+1]$; Anal. calcd. for $\mathrm{C}_{19} \mathrm{H}_{14} \mathrm{BrN}_{5} \mathrm{O}_{2} \mathrm{~S}: \mathrm{C}, 50.01 ; \mathrm{H}, 3.09 ; \mathrm{N}$, 15.35; S, 7.03; Found: C, 50.02; H, 3.09; N, 15.36; S, 7.03.

$\mathrm{N}$-(2-Fluorophenyl)-2-[(10-bromo-3-(4'-methoxyphenyl)-2-oxo-2H-[1,2,4]triazino[2,3-c] quinazolin-6-yl) thio]acetamide (4.24). Yield - 87.7\%. M.p. $-284-286^{\circ} \mathrm{C}$; ${ }^{1} \mathrm{H}$ NMR $\delta: 3.91$ (s, 3H, $\left.-\mathrm{OCH}_{3}\right), 4.26$ (s, 2H, - $\left.\mathrm{SCH}_{2}\right), 7.27-$ 7.08 (m, 5H, 3-PhH-3', 5', 6-Ph H-3', 4', 5'), 7.70 (d, $J=8.8 \mathrm{~Hz}$, 1H, 6-Ph H-6'), 8.10-7.88 (m, 2H, H-8, 9), 8.43 (d, J=8.7 Hz, 2H, 3-Ph H-2', 6'), 8.61 (s, 1H, H-11), 10.00 (s, 1H, NH); LC$\mathrm{MS}, \mathrm{m} / \mathrm{z}=567[\mathrm{M}+1]$; Anal. calcd. for $\mathrm{C}_{25} \mathrm{H}_{17} \mathrm{BrFN}_{5} \mathrm{O}_{3} \mathrm{~S}: \mathrm{C}$, 53.01; H, 3.03; Br, 14.11; F, 3.35; N, 12.36; S, 5.66; Found: C, 53.04; H, 3.03; Br, 14.11; F, 3.35; N, 12.34; S, 5.65.

$N$-(3-Fluorophenyl-)2-[(10-bromo-3-(4'-methoxyphenyl)-2-oxo-2H-[1,2,4] triazino[2,3-c]quinazolin-6-yl) thio]acetamide (4.25). Yield - 81.7\%. M.p. $-288-290^{\circ} \mathrm{C}$; ${ }^{1} \mathrm{H}$ NMR $\delta: 3.91$ (s, 3H, $\left.-\mathrm{OCH}_{3}\right), 4.21$ (s, 2H, $\left.-\mathrm{SCH}_{2}\right), 6.80$ (dd, $J=9.4,7.8 \mathrm{~Hz}, 1 \mathrm{H}, 6-\mathrm{Ph} \mathrm{H}-4$ ), 7.09 (d, $J=7.6 \mathrm{~Hz}, 2 \mathrm{H}, 3-\mathrm{Ph}$ H-2', 5'), 7.42-7.23 (m, 2H, 6-Ph H-5', 6'), 7.59 (dd, $J=11.3$, $\left.0.7 \mathrm{~Hz}, 1 \mathrm{H}, 6-\mathrm{PhH}-2^{\prime}\right), 7.67$ (d, $\left.J=7.8 \mathrm{~Hz}, 1 \mathrm{H}, \mathrm{H}-8\right), 8.01$ (d, $J$ $=7.8 \mathrm{~Hz}, 1 \mathrm{H}, \mathrm{H}-9), 8.43$ (d, $\left.J=8.0 \mathrm{~Hz}, 2 \mathrm{H}, 3-\mathrm{Ph} \mathrm{H}-2^{\prime}, 6^{\prime}\right), 8.53$ (s, 1H, H-11), 10.53 (s, 1H, NH); LC-MS, m/z = 568 [M+2]; Anal. calcd. for $\mathrm{C}_{25} \mathrm{H}_{17} \mathrm{BrFN}_{5} \mathrm{O}_{3} \mathrm{~S}: \mathrm{C}, 53.01 ; \mathrm{H}, 3.03 ; \mathrm{Br}$, 14.11; F, 3.35; N, 12.36; S, 5.66; Found: C, 53.04; H, 3.03; Br, 14.11; F, 3.35; N, 12.34; S, 5.65.

2-[(10-Bromo-3-(4'-fluorophenyl)-2-oxo-2H$[1,2,4]$ triazino[2,3-c] quinazolin-6-yl)thio]acetamide (4.26). Yield - 66.7\%. M.p. $-289-291^{\circ} \mathrm{C}$; LC-MS, m/z = 463 $[\mathrm{M}+3]$; Anal. calcd. for $\mathrm{C}_{18} \mathrm{H}_{11} \mathrm{BrFN}_{5} \mathrm{O}_{2} \mathrm{~S}$ : C, 46.97; H, 2.41; N, 15.22; S, 6.97; Found: C, 46.96; H, 2.41; N, 15.22; S, 6.98.

1.4. General procedure for the synthesis of $2-\left[\left(8-R_{1^{-}}\right.\right.$ $9-R_{2}-10-R_{3}-3-R-2-o x o-2 H$ - $[1,2,4]$ triazino[2,3-c]quinazoline-6-yl)thio]acetonitriles (5.1-5.18).

Method A. Add $0.75 \mathrm{~g}(10 \mathrm{mmol})$ of chloroacetonitrile to the suspension of the proper potassium $8-\mathrm{R}_{1}-9$ $\mathrm{R}_{2}$-10-R $-\mathrm{R}_{3}$-3-R-2-oxo-2H-[1,2,4]triazino[2,3-c]guinazoline-6-thiolates $(2.1-2.18)(10 \mathrm{mmol})$ in $20 \mathrm{ml}$ of propanol-2 and reflux for 1-1.5 hours. Cool the resulted mixture, filter the solid and dry. Crystallize the compounds from DMF-water.

Method B. Stir the mixture of $10 \mathrm{mmol}$ of the proper 2-[(8-R $-9-\mathrm{R}_{2}-10-\mathrm{R}_{3}-3-\mathrm{R}-2-$ oxo- $2 \mathrm{H}$ - $[1,2,4]$ triazino [2,3-c] quinazoline-6-yl)thio]acetamides $(4.2,4.5,4.8,4.12)$, $0.35 \mathrm{~g}(0.6 \mathrm{mmol}) \mathrm{NaCl}, 25 \mathrm{ml}$ anhydrous dichloroethane, $1.0 \mathrm{~g}$ (6.5 mmol) phosphorous oxychloride and 1-2 drops of pyridine when heating $\left(84^{\circ} \mathrm{C}\right)$ for $50 \mathrm{~min}$. Then increase the temperature to $88^{\circ} \mathrm{C}$ and continue to stir for 4 hours. Evaporate the solvent under vacuum, recrystallize the solid obtained from DMA-water.

2-[(3-Methyl-2-oxo-2H-[1,2,4]triazino[2,3-c]quinazoline-6-yl)thio]acetonitrile (5.1). Yield-99.3\% (Method A). M.p. $-279-281^{\circ} \mathrm{C} ;{ }^{1} \mathrm{H}$ NMR $\delta: 2.44$ (s, 3H, $\left.\mathrm{CH}_{3}\right), 4.28$ (s, 2H, $\left.-\mathrm{SCH}_{2}\right), 7.68$ (t, $\left.J=7.4 \mathrm{~Hz}, 1 \mathrm{H}, \mathrm{H}-10\right), 7.86(\mathrm{~d}, J=8.2 \mathrm{~Hz}, 1 \mathrm{H}$, $\mathrm{H}-8), 7.97$ (t, $J=8.2 \mathrm{~Hz}, 1 \mathrm{H}, \mathrm{H}-9), 8.56$ (d, $J=7.8 \mathrm{~Hz}, 1 \mathrm{H}$, H-11); LC-MS, m/z = 284 [M+1], 286 [M+3]; Anal. calcd. for $\mathrm{C}_{13} \mathrm{H}_{9} \mathrm{~N}_{5} \mathrm{OS}$ : C, 55.11; H, 3.20; N, 24.72; S, 11.32; Found: C, 55.10; H, 3.20; N, 24.72; S, 11.33.

2-[(3-Phenyl-2-oxo-2H-[1,2,4]triazino[2,3-c]quinazolin-6-yl)thio]acetonitrile (5.2). Yield-99.6\% (Method A);

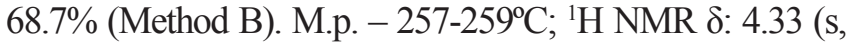
2H, $-\mathrm{SCH}_{2}$ ), 7.63-7.48 (m, 3H, 3-Ph H-3', 4', 5'), 7.70 (t, $J=$ $7.5 \mathrm{~Hz}, 1 \mathrm{H}, \mathrm{H}-10), 7.87(\mathrm{~d}, J=7.8 \mathrm{~Hz}, 1 \mathrm{H}, \mathrm{H}-8), 7.98(\mathrm{t}, J=7.4 \mathrm{~Hz}$, 1H, H-9), 8.32 (d, $J=7.8 \mathrm{~Hz}, 2 \mathrm{H}, 3-\mathrm{Ph} \mathrm{H}-2^{\prime}, 6^{\prime}$ ), 8.58 (d, $J$ $=7.8 \mathrm{~Hz}, 1 \mathrm{H}, \mathrm{H}-11)$; LC-MS, m/z = $346[\mathrm{M}+1], 348$ [M+3]; Anal. calcd. for $\mathrm{C}_{18} \mathrm{H}_{11} \mathrm{~N}_{5} \mathrm{OS}$ : C, 62.60; H, 3.21; N, 20.28; S, 9.28; Found: C, 62.62; H, 3.20; N, 20.28; S, 9.27.

2-[(3-(3',4'-Dimethylphenyl)-2-oxo-2H-[1,2,4]triazino[2,3-c] quinazolin-6-yl)thio]acetonitrile (5.3). Yield - 99.7\% (Method A). M.p. - 258-261 ${ }^{\circ} \mathrm{C} ;{ }^{1} \mathrm{H}$ NMR $\delta: 2.35$ (s, $\left.3 \mathrm{H}, 3-\mathrm{Ph} 3-\mathrm{CH}_{3}\right), 2.38$ (s, 3H, 3-Ph 4- $\left.\mathrm{CH}_{3}\right), 4.31$ (s, 2H, $-\mathrm{SCH}_{2}$ ), 7.26 (d, $\left.J=7.9 \mathrm{~Hz}, 1 \mathrm{H}, 3-\mathrm{Ph} \mathrm{H}-5^{\prime}\right), 7.70$ (t, $J=7.4 \mathrm{~Hz}, 1 \mathrm{H}$, H-10), 7.87 (d, $J=8.0 \mathrm{~Hz}, 1 \mathrm{H}, \mathrm{H}-8), 7.97$ (t, $J=7.4 \mathrm{~Hz}, 1 \mathrm{H}$, H-9), 8.05 (d, $J=7.8$ Hz, 1H, 3-Ph H-6'), 8.10 (s, 1H, 3-Ph H-2'), 8.57 (d, J=7.8 Hz, 1H, H-11); LC-MS, m/z =374 [M+1], 376 [M+3]; Anal. calcd. for $\mathrm{C}_{20} \mathrm{H}_{15} \mathrm{~N}_{5} \mathrm{OS}$ : C, 64.33; H, 4.05; N, 18.75; S, 8.59; Found: C, 64.34; H, 4.05; N, 18.74; S, 8.59.

2-[(3-(4'-i-Propylphenyl)-2-oxo-2H-[1,2,4]triazino[2,3-c]quinazolin-6-yl)thio]acetonitrile (5.4). Yield 99.9\% (Method A). M.p. $-176-178^{\circ} \mathrm{C} ;{ }^{1} \mathrm{HNMR} \delta: 1.32(\mathrm{~d}, J=$ $\left.5.5 \mathrm{~Hz}, 6 \mathrm{H},-\mathrm{CH}\left(\underline{\mathrm{CH}}_{3}\right)_{2}\right), 2.59-2.53\left(\mathrm{~m}, 1 \mathrm{H},-\underline{\mathrm{CH}}\left(\mathrm{CH}_{3}\right)_{2}\right), 4.32$ (s, 2H, $-\mathrm{SCH}_{2}$ ), 7.37 (d, $\left.J=7.8 \mathrm{~Hz}, 2 \mathrm{H}, 3-\mathrm{Ph} \mathrm{H}-3^{\prime}, 5^{\prime}\right), 7.69$ (t, $J=7.3 \mathrm{~Hz}, 1 \mathrm{H}, \mathrm{H}-10), 7.87$ (d, $J=7.9 \mathrm{~Hz}, 1 \mathrm{H}, \mathrm{H}-8), 7.97$ (t, $J=7.2 \mathrm{~Hz}, 1 \mathrm{H}, \mathrm{H}-9), 8.25$ (d, $J=7.7 \mathrm{~Hz}, 2 \mathrm{H}, 3-\mathrm{Ph} \mathrm{H}-2^{\prime}$, $\left.6^{\prime}\right), 8.57$ (d, J=7.7 Hz, 1H, H-11); LC-MS, m/z =388 [M+1], $390[\mathrm{M}+3]$; Anal. calcd. for $\mathrm{C}_{21} \mathrm{H}_{17} \mathrm{~N}_{5} \mathrm{OS}$ : C, 65.10; H, 4.42; N, 18.08; S, 8.28; Found: C, 65.10; H, 4.41; N, 18.09; S, 8.28.

2-[(3-(4'-(tert-Butyl)phenyl)-2-oxo-2H-[1,2,4]triazino[2,3-c] quinazolin-6-yl)thio] acetonitrile (5.5). Yield - 96.0\% (Method A); 73.7\% (Method B). M.p. - 256-258 C; ${ }^{1} \mathrm{H}$ NMR $\delta: 1.39$ (s, 9H, -C( $\left.\left.\underline{\mathrm{CH}}_{3}\right)_{3}\right)$; 4.32 (s, 2H, - $\left.\mathrm{SCH}_{2}\right), 7.53$ (d, $\left.J=8.0 \mathrm{~Hz}, 2 \mathrm{H}, 3-\mathrm{Ph} \mathrm{H}-3^{\prime}, 5^{\prime}\right), 7.69$ (t, $J=7.4 \mathrm{~Hz}, 1 \mathrm{H}$, H-10), 7.86 (d, $J=7.9 \mathrm{~Hz}, 1 \mathrm{H}, \mathrm{H}-8), 7.97$ (t, $J=7.4 \mathrm{~Hz}, 1 \mathrm{H}$, H-9), 8.26(d, $\left.J=8.1 \mathrm{~Hz}, 2 \mathrm{H}, 3-\mathrm{PhH}-2^{\prime}, 6^{\prime}\right), 8.57$ (d, $J=7.9 \mathrm{~Hz}$, 1H, H-11); LC-MS, m/z = 402 [M+1], 404 [M+3]; Anal. calcd. for $\mathrm{C}_{22} \mathrm{H}_{19} \mathrm{~N}_{5} \mathrm{OS}$ : C, 65.81; H, 4.77; N, 17.44; O, 3.99; S, 7.99; Found: C, 65.80; H, 4.77; N, 17.44; O, 3.99; S, 8.00.

2-[(3-(4'-Methoxyphenyl)-2-oxo-2H-[1,2,4]triazino[2,3-c]quinazolin-6-yl)thio]acetonitrile (5.6). Yield 82.0\% (Method A). M.p. $-272-274^{\circ} \mathrm{C} ;{ }^{1} \mathrm{H}$ NMR $\delta: 3.90$ (s, $\left.3 \mathrm{H},-\mathrm{OCH}_{3}\right), 4.32\left(\mathrm{~s}, 2 \mathrm{H},-\mathrm{SCH}_{2}\right), 7.05(\mathrm{~d}, J=7.3 \mathrm{~Hz}, 2 \mathrm{H}, 3-\mathrm{Ph}$ H-3', 5'), 7.70 (t, $J=7.6$ Hz, 1H, H-10), 7.88 (d, $J=7.6$ Hz, $1 \mathrm{H}, \mathrm{H}-8), 7.98$ (d, $J=7.6 \mathrm{~Hz}, 1 \mathrm{H}, \mathrm{H}-9), 8.39$ (d, $J=7.6 \mathrm{~Hz}$, 2H, 3-Ph H-2', 6'), 8.58 (d, J=7.4 Hz, 1H, H-11); LC-MS, $\mathrm{m} / \mathrm{z}=376[\mathrm{M}+1]$, $378[\mathrm{M}+3]$; Anal. calcd. for $\mathrm{C}_{19} \mathrm{H}_{13} \mathrm{~N}_{5} \mathrm{O}_{2} \mathrm{~S}$ : C, 60.79; H, 3.49; N, 18.66; O, 8.52; S, 8.54; Found: C, 60.80; H, 3.49; N, 18.66; O, 8.52; S, 8.53. 
2-[(3-(4'-Ethoxyphenyl)-2-oxo-2 $H$ - $[1,2,4]$ triazino [2,3-c] quinazolin-6-yl)thio]acetonitrile (5.7). Yield - 99.9\% (Method A). M.p. $-268-270^{\circ} \mathrm{C} ;{ }^{1} \mathrm{H}$ NMR $\delta: 1.45$ (t, $J=5.6 \mathrm{~Hz}$, $\left.3 \mathrm{H},-\mathrm{OCH}_{2} \underline{\mathrm{CH}}_{3}\right), 4.15$ (dd, $\left.J=11.1,5.4 \mathrm{~Hz}, 2 \mathrm{H},-\mathrm{OCH}_{2} \mathrm{CH}_{3}\right)$, $4.32\left(\mathrm{~s}, 2 \mathrm{H},-\mathrm{SCH}_{2}\right), 7.02\left(\mathrm{~d}, J=7.6 \mathrm{~Hz}, 2 \mathrm{H}, 3-\mathrm{Ph} \mathrm{H}-33^{\prime}, 5^{\prime}\right)$, 7.71 (t, $J=7.3 \mathrm{~Hz}, 1 \mathrm{H}, \mathrm{H}-10), 7.88$ (d, $J=7.3 \mathrm{~Hz}, 1 \mathrm{H}, \mathrm{H}-8)$, 7.98 (t, $J=7.3 \mathrm{~Hz}, 1 \mathrm{H}, \mathrm{H}-9), 8.38$ (d, $J=7.7 \mathrm{~Hz}, 2 \mathrm{H}, 3-\mathrm{Ph} \mathrm{H}-2^{\prime}$, $\left.6^{\prime}\right), 8.58(\mathrm{~d}, J=7.3 \mathrm{~Hz}, 1 \mathrm{H}, \mathrm{H}-11)$; LC-MS, m/z = 390 [M+1], $392[\mathrm{M}+3]$; Anal. calcd. for $\mathrm{C}_{20} \mathrm{H}_{15} \mathrm{~N}_{5} \mathrm{O}_{2} \mathrm{~S}: \mathrm{C}, 61.68 ; \mathrm{H}, 3.88$; N, 17.98; S, 8.23; Found: C, 61.67; H, 3.88; N, 17.98; S, 8.24.

2-[(3-(4'-Fluorophenyl)-2-oxo-2H-[1,2,4]triazino [2,3-c] quinazolin-6-yl)thio]acetonitrile (5.8). Yield - 99.9\% (Method A); 81.3\% (Method B). M.p. $-266-268^{\circ} \mathrm{C} ;{ }^{1} \mathrm{H}$ NMR

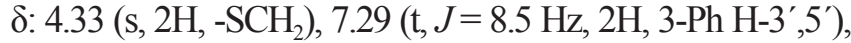
$7.71(\mathrm{t}, J=7.4 \mathrm{~Hz}, 1 \mathrm{H}, \mathrm{H}-10), 7.89$ (d, $J=8.0 \mathrm{~Hz}, 1 \mathrm{H}, \mathrm{H}-8)$, 7.99 (t, $J=7.3 \mathrm{~Hz}, 1 \mathrm{H}, \mathrm{H}-9), 8.43$ (dd, $J=7.5,5.9 \mathrm{~Hz}, 2 \mathrm{H}, 3-\mathrm{Ph}$ H-2', 6'), 8.59 (d, J=7.9 Hz, 1H, H-11); LC-MS, m/z = 364 $[\mathrm{M}+1], 366[\mathrm{M}+3]$; Anal. calcd. for $\mathrm{C}_{18} \mathrm{H}_{10} \mathrm{FN}_{5} \mathrm{OS}: \mathrm{C}, 59.50 ; \mathrm{H}$, 2.77; N, 19.27; S, 8.82; Found:C, 59.51; H, 2.77; N, 19.27; S, 8.81.

2-[(8-Methyl-3-phenyl-2-oxo-2H-[1,2,4]triazino [2,3-c] quinazolin-6-yl)thio]acetonitrile (5.9). Yield - 98.67\% (MethodA). M.p. $-255-257^{\circ} \mathrm{C} ;{ }^{1} \mathrm{HNMR} \delta: 2.76\left(\mathrm{~s}, 3 \mathrm{H},-\mathrm{CH}_{3}\right)$, 4.32 (s, 2H, - $\mathrm{SCH}_{2}$ ), 7.63-7.46 (m, 4H, H-10, 3-Ph H-3', 4', 5'), 7.81 (d, $J=5.6 \mathrm{~Hz}, 1 \mathrm{H}, \mathrm{H}-9), 8.31$ (d, $J=8.8 \mathrm{~Hz}, 2 \mathrm{H}, 3-\mathrm{Ph} \mathrm{H}-2^{\prime}$, 6'), 8.41 (d, $J=7.8 \mathrm{~Hz}, 1 \mathrm{H}, \mathrm{H}-11)$; LC-MS, m/z = 360 [M+1], 362 [M+3]; Anal. calcd. for $\mathrm{C}_{19} \mathrm{H}_{13} \mathrm{~N}_{5} \mathrm{OS}$ : C, 63.49; H, 3.65; N, 19.49; S, 8.92; Found: C, 63.48; H, 3.65; N, 19.49; S, 8.93.

2-[(9-Fluoro-3-phenyl-2-oxo-2H-[1,2,4]triazino [2,3-c]quinazolin-6-yl)thio]acetonitrile (5.10). Yield - 88.0\% (Method A); 71.9\% (Method B). M.p. $-250-253^{\circ} \mathrm{C} ;{ }^{1} \mathrm{HNMR}$ $\delta: 4.33\left(\mathrm{~s}, 2 \mathrm{H},-\mathrm{SCH}_{2}\right), 7.63-7.45(\mathrm{~m}, J=14.9,7.7 \mathrm{~Hz}, 5 \mathrm{H}, \mathrm{H}-8$, 10, 3-Ph H-3', $4^{\prime}, 5^{\prime}$ ), 8.29 (d, $J=7.2$ Hz, 2H, 3-Ph H-2',6'), $8.64(\mathrm{dd}, J=8.7,5.9 \mathrm{~Hz}, 1 \mathrm{H}, \mathrm{H}-11)$; LC-MS, m/z $=364$ [M+1], $366[\mathrm{M}+3]$; Anal. calcd. for $\mathrm{C}_{18} \mathrm{H}_{10} \mathrm{FN}_{5} \mathrm{OS}$ : C, 59.50; H, 2.77; N, 19.27; S, 8.82; Found: C, 59.51; H, 2.77; N, 19.26; S, 8.82.

2-[(9-Fluoro-3-(4'-methoxyphenyl)-2-oxo-2H$[1,2,4]$ triazino[2,3-c] quinazolin-6-yl)thio] acetonitrile (5.11). Yield - 74.67\% (Method A). M.p. - 273-275 C; ${ }^{1} \mathrm{H}$ NMR $\delta$ : $3.90\left(\mathrm{~s}, 3 \mathrm{H},-\mathrm{CH}_{3}\right), 4.32\left(\mathrm{~s}, 2 \mathrm{H},-\mathrm{SCH}_{2}\right), 7.04(\mathrm{~d}, J=8.2 \mathrm{~Hz}, 2 \mathrm{H}$, 3-Ph H-3',5'), 7.50 (dd, $J=8.6,6.6 \mathrm{~Hz}, 1 \mathrm{H}, \mathrm{H}-10), 7.57$ (d, $J=$ $7.6 \mathrm{~Hz}, 1 \mathrm{H}, \mathrm{H}-8$ ), 8.36 (d, J=8.3 Hz, 2H, 3-Ph H-2',6'), 8.63 (dd, $J=8.7,5.9 \mathrm{~Hz}, 1 \mathrm{H}, \mathrm{H}-11)$; LC-MS, m/z = 394 [M+1], $396[\mathrm{M}+3]$; Anal. calcd. for $\mathrm{C}_{19} \mathrm{H}_{12} \mathrm{FN}_{5} \mathrm{O}_{2} \mathrm{~S}: \mathrm{C}, 58.01 ; \mathrm{H}, 3.07$; N, 17.80; S, 8.15; Found: C, 58.02; H, 3.07; N, 17.80; S, 8.14.

2-[(9-Fluoro-3-(4'-fluorophenyl)-2-oxo-2 $H$ - $[1,2,4]$ triazino[2,3-c] quinazolin-6-yl)thio] acetonitrile (5.12). Yield - 48.0\% (Method A). M.p. $-258-260^{\circ} \mathrm{C}$; ${ }^{1} \mathrm{H}$ NMR $\delta$ : 4.33 ( $\left.\mathrm{s}, 2 \mathrm{H},-\mathrm{SCH}_{2}\right), 7.29$ (t, $\left.J=8.4 \mathrm{~Hz}, 2 \mathrm{H}, 3-\mathrm{Ph} \mathrm{H}-3^{\prime}, 5^{\prime}\right), 7.51$ (t, $J=7.5 \mathrm{~Hz}, 1 \mathrm{H}, \mathrm{H}-10), 7.58$ (d, $J=9.2 \mathrm{~Hz}, 1 \mathrm{H}, \mathrm{H}-8), 8.40$ (t, $\left.J=5.7 \mathrm{~Hz}, 2 \mathrm{H}, 3-\mathrm{Ph} \mathrm{H}-2^{\prime}, 6^{\prime}\right), 8.63$ (dd, $J=8.3,6.0 \mathrm{~Hz}, 1 \mathrm{H}$, H-11); LC-MS, m/z = 382 [M+1], 384 [M+3]; Anal. calcd. for $\mathrm{C}_{18} \mathrm{H}_{9} \mathrm{~F}_{2} \mathrm{~N}_{5} \mathrm{OS}$ : C, 56.69; H, 2.38; N, 18.36; S, 8.41; Found: C, $56.68 ; \mathrm{H}, 2.38 ; \mathrm{N}, 18.37$; $\mathrm{S}, 8.41$.

2-[(10-Chloro-3-phenyl-2-oxo-2 $H$ - $[1,2,4]$ triazino [2,3-c]quinazolin-6-yl)thio]acetonitrile (5.13). Yield -

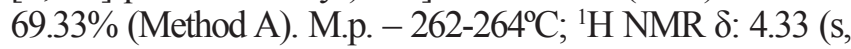
2H, $-\mathrm{SCH}_{2}$ ), 7.65-7.37 (m, 3H, 3-Ph H-3', 4', 5'), 7.88 (d, J $=8.6 \mathrm{~Hz}, 1 \mathrm{H}, \mathrm{H}-8), 7.95(\mathrm{~d}, J=8.6 \mathrm{~Hz}, 1 \mathrm{H}, \mathrm{H}-9), 8.31(\mathrm{~d}$, $J=7.3 \mathrm{~Hz}, 2 \mathrm{H}, 3-\mathrm{Ph} \mathrm{H}-2^{\prime}, 6^{\prime}$ ), 8.50 (s, 1H, H-11); LC-MS, $\mathrm{m} / \mathrm{z}=380[\mathrm{M}+1], 382[\mathrm{M}+3], 383[\mathrm{M}+4]$; Anal. calcd. for
$\mathrm{C}_{18} \mathrm{H}_{10} \mathrm{CIN}_{5} \mathrm{OS}$ : C, 56.92; H, 2.65; N, 18.44; S, 8.44; Found: C, 56.92; H, 2.65; N, 18.43; S, 8.45.

2-[(10-Chloro-3-(4'-methylphenyl)-2-oxo-2 $H$ - $[1,2,4]$ triazino[2,3-c] quinazolin-6-yl)thio] acetonitrile (5.14). Yield - 64.0\% (Method A). M.p. $-250-253^{\circ} \mathrm{C} ;{ }^{1} \mathrm{H}$ NMR $\delta$ : 2.46 (s, 3H, $\left.-\mathrm{CH}_{3}\right), 4.32\left(\mathrm{~s}, 2 \mathrm{H}, \mathrm{S}-\mathrm{CH}_{2}-\right), 7.33$ (d, J=7.8 Hz, $\left.2 \mathrm{H}, 3-\mathrm{PhH}-3^{\prime}, 5^{\prime}\right), 7.87$ (d, $\left.J=8.6 \mathrm{~Hz}, 1 \mathrm{H}, \mathrm{H}-8\right), 7.93$ (d, $J=8.6$ Hz, 1H, H-9), 8.24 (d, J=7.8 Hz, 2H, 3-Ph H-2', 6'), 8.49 (s, 1H, H-11); Anal. calcd. for $\mathrm{C}_{19} \mathrm{H}_{12} \mathrm{ClN}_{5} \mathrm{OS}$ : C, 57.94; H, 3.07; N, 17.78; S, 8.14; Found: C, 57.93; H, 3.07; N, 17.79; S, 8.14.

2-[(10-Chloro-3-(4'-fluorophenyl)-2-oxo-2H-[1,2,4] triazino[2,3-c]quinazolin-6-yl)thio]acetonitrile (5.15). Yield - 69.33\% (Method A). M.p. $-270-272^{\circ} \mathrm{C}$; ${ }^{1} \mathrm{H}$ NMR $\delta$ : 4.33 (s, 2H, -S-CH $\left.2^{-}\right), 7.29$ (t, $J=8.6$ Hz, 2H, 3-Ph H-3', 5'), 7.88 (d, $J=8.7 \mathrm{~Hz}, 1 \mathrm{H}, \mathrm{H}-8), 7.95$ (d, $J=8.5 \mathrm{~Hz}, 1 \mathrm{H}, \mathrm{H}-9)$, $8.42\left(\mathrm{dd}, J=8.0,5.8 \mathrm{~Hz}, 2 \mathrm{H}, 3-\mathrm{Ph} \mathrm{H}-2^{\prime}, 6^{\prime}\right), 8.49$ (s, 1H, H-11); LC-MS, m/z = 398 [M+1], 400 [M+3], 401 [M+4]; Anal. calcd. for $\mathrm{C}_{18} \mathrm{H}_{9} \mathrm{ClFN}_{5} \mathrm{OS}$ : C, 54.35; H, 2.28; N, 17.60; S, 8.06; Found: C, 54.36; H, 2.27; N, 17.60; S, 8.06.

2-[(10-Bromo-3-phenyl-2-oxo-2H-[1,2,4] triazino [2,3-c]quinazolin-6-yl)thio] acetonitrile (5.16). Yield 96.7\% (Method A). M.p. - 260-252 ${ }^{\circ} \mathrm{C}$; ${ }^{1} \mathrm{H}$ NMR $\delta: 4.33$ (s, 2H, $-\mathrm{SCH}_{2}$ ), 7.66-7.41 (m, 3H, 3-Ph H-3', 4', 5'), 7.81 (d, $J$ $=8.6 \mathrm{~Hz}, 1 \mathrm{H}, \mathrm{H}-8), 8.08(\mathrm{~d}, J=8.6 \mathrm{~Hz}, 1 \mathrm{H}, \mathrm{H}-9), 8.31(\mathrm{~d}, J=$ $7.3 \mathrm{~Hz}, 2 \mathrm{H}, 3-\mathrm{Ph} \mathrm{H}-2^{\prime}, 6^{\prime}$ ), 8.65 (s, 1H, H-11); LC-MS, m/z = $425[\mathrm{M}+1]$; Anal. calcd. for $\mathrm{C}_{18} \mathrm{H}_{10} \mathrm{BrN}_{5} \mathrm{OS}$ : C, 50.96; $\mathrm{H}, 2.38$; N, 16.51; S, 7.56; Found: C, 50.97; H, 2.38; N, 16.50; S, 7.56.

2-[(10-Bromo-3-(4'-methylphenyl)-2-oxo-2 $H$ - $[1,2,4]$ triazino[2,3-c] quinazolin-6-yl)thio]acetonitrile (5.17). Yield $-77.2 \%$ (Method A). M.p. - 268-270 C; ${ }^{1} \mathrm{H}$ NMR $\delta: 2.46$ (s, $\left.3 \mathrm{H},-\mathrm{CH}_{3}\right), 4.32\left(\mathrm{~s}, 2 \mathrm{H},-\mathrm{SCH}_{2}\right), 6.32(\mathrm{~d}, J=7.9 \mathrm{~Hz}, 2 \mathrm{H}, 3-\mathrm{Ph}$ $\left.\mathrm{H}^{-3} 3^{\prime}, 5^{\prime}\right), 7.80(\mathrm{~d}, J=8.6 \mathrm{~Hz}, 1 \mathrm{H}, \mathrm{H}-8), 8.06(\mathrm{dd}, J=8.6,1.6 \mathrm{~Hz}$, 1H, H-9), 8.24 (d, $J=8.0$ Hz, 2H, 3-Ph H-2', 6'), 8.63 (s, 1H, H-11); LC-MS, m/z = 439 [M+1]; Anal. calcd. for $\mathrm{C}_{19} \mathrm{H}_{12} \mathrm{BrN}_{5} \mathrm{OS}$ : C, 52.07; H, 2.76; N, 15.98; S, 7.32; Found: C, 52.06; H, 2.76; N, 15.98; S, 7.33.

2-[(10-Bromo-3-(4'-methoxyphenyl)-2-oxo- $2 \mathrm{H}$ $[1,2,4]$ triazino[2,3-c]quinazolin-6-yl)thio]acetonitrile (5.18). Yield - 88.0\% (Method A). M.p. $-266-268^{\circ} \mathrm{C} ;{ }^{1} \mathrm{H}$ NMR $\delta$ : 3.88(s, 3H, $\left.-\mathrm{CH}_{3}\right), 4.30$ (s, 2H, $\left.-\mathrm{SCH}_{2}\right), 7.03(\mathrm{~d}, 2 \mathrm{H}, 3-\mathrm{Ph} \mathrm{H}-3$ ', $\left.5^{\prime}\right), 7.81(\mathrm{~d}, 1 \mathrm{H}, \mathrm{H}-8), 8.04$ (d, 1H, H-9), 8.37 (d, 2H, 3-Ph H-2', 6'), 8.62 (s, 1H, H-11); LC-MS, m/z = 455 [M+1], $456[\mathrm{M}+2]$, $458[\mathrm{M}+4]$; Anal. calcd. for $\mathrm{C}_{19} \mathrm{H}_{12} \mathrm{BrN}_{5} \mathrm{O}_{2} \mathrm{~S}: \mathrm{C}, 50.23 ; \mathrm{H}, 2.66$; N, 15.42; S, 7.06; Found: C, 50.22; H, 2.66; N, 15.43; S, 7.06.

2-[(10-Bromo-3-(4'-fluorophenyl)-2-oxo-2H-[1,2,4] triazino[2,3-c] quinazolin-6-yl)thio]acetonitrile (5.19). Yield $-64.0 \%$. M.p. $-259-261^{\circ} \mathrm{C} ;{ }^{1} \mathrm{H}$ NMR $\delta: 4.33$ (s, 2H, $-\mathrm{SCH}_{2}$ ), 7.29 (t, $\left.J=8.4 \mathrm{~Hz}, 3 \mathrm{H}, 3-\mathrm{Ph} \mathrm{H}-3^{\prime}, 5^{\prime}\right), 7.81$ (d, $J=8.6 \mathrm{~Hz}, 1 \mathrm{H}$, $\mathrm{H}-8), 8.07$ (d, $J=8.3 \mathrm{~Hz}, 1 \mathrm{H}, \mathrm{H}-9), 8.42$ (dd, $J=7.3,5.8 \mathrm{~Hz}$, 2H, 3-PhH-2', 6'), 8.64 (s, 1H, H-11); LC-MS, m/z = 446 [M+4]; Anal. calcd. for $\mathrm{C}_{18} \mathrm{H}_{9} \mathrm{BrFN}_{5} \mathrm{OS}$ : C, 48.88; H, 2.05; N, 15.84; $\mathrm{S}$, 7.25; Found: C, 48.89; H, 2.05; N, 15.84; S, 7.24.

\section{Pharmacology}

Antimicrobial and antifungal test

Sensitivity of microorganisms to the compounds synthesized was assessed according to the methods described [9]. The assay was conducted on the Mueller-Hinton medium by two-fold serial dilution of compounds in $1 \mathrm{ml}$. After that $0.1 \mathrm{ml}$ of microbial seeding (106 cells $/ \mathrm{ml})$ was added. The minimal inhibitory concentration of com- 

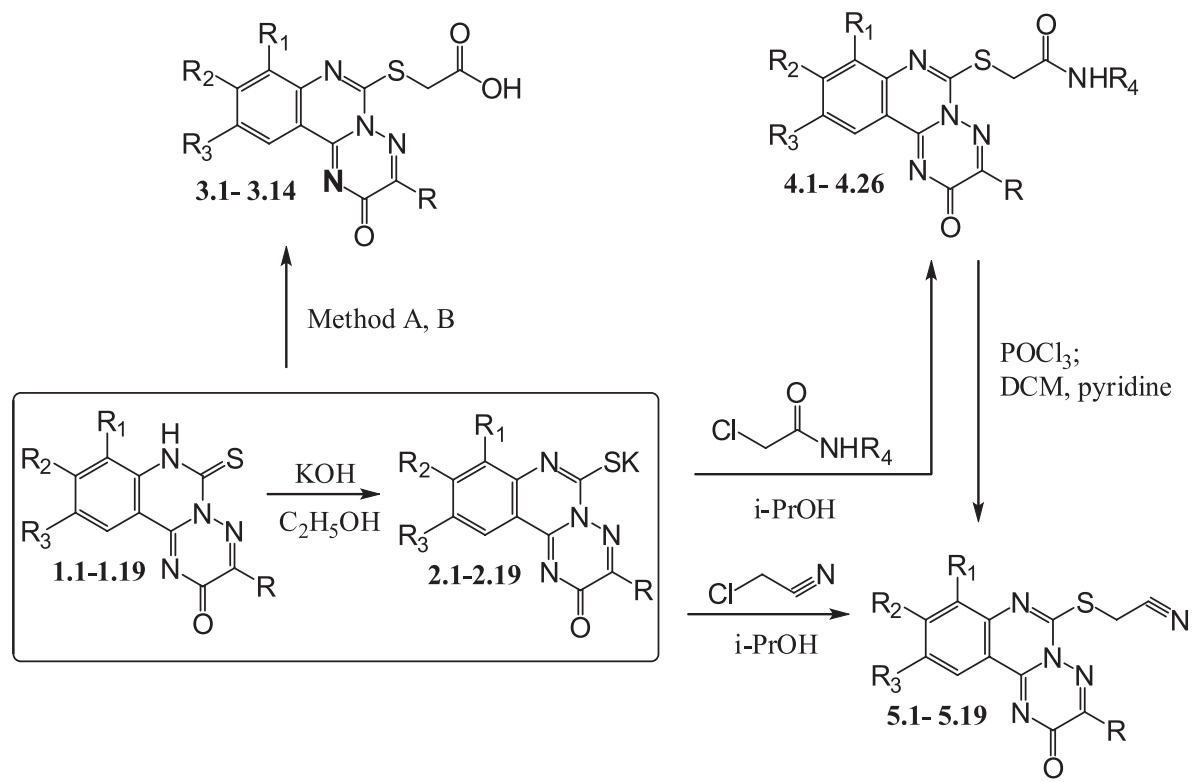

Method A: $\mathrm{ClCH}_{2} \mathrm{COOH}, \mathrm{NaOH}, \mathrm{H}_{2} \mathrm{O}$; Method B: $\mathrm{ClCH}_{2} \mathrm{COOH}, \mathrm{Na}, \mathrm{C}_{2} \mathrm{H}_{5} \mathrm{OH} ; \mathrm{R}=\mathrm{C}_{2} \mathrm{H}_{5} \mathrm{OH} ; \mathrm{R}=\mathrm{CH}_{3}$, $\mathrm{Ph}, 4-\mathrm{CH}_{3} \mathrm{Ph}, 3,4-\left(\mathrm{CH}_{3}\right)_{2} \mathrm{Ph}, 4-\mathrm{C}_{2} \mathrm{H}_{5} \mathrm{Ph}, 4-\left(\mathrm{CH}_{3}\right)_{2} \mathrm{CHPh}, 4-\left(\mathrm{CH}_{3}\right)_{3} \mathrm{CPh}, 4-\mathrm{CH}_{3} \mathrm{OPh}, 4-\mathrm{C}_{2} \mathrm{H}_{5} \mathrm{OPh}$, 4- $\mathrm{FPh} ; \mathrm{R}_{1}=\mathrm{H}, \mathrm{CH}_{3}, \mathrm{Br} ; \mathrm{R}_{2}=\mathrm{H}, \mathrm{F}, \mathrm{Br} ; \mathrm{R}_{3}=\mathrm{H}, \mathrm{Cl}, \mathrm{Br} ; \mathrm{R}_{4}=\mathrm{H}, 2-\mathrm{FPh}, 3-\mathrm{FPh}, 4-\mathrm{BrPh}, 4-\mathrm{CH}_{3} \mathrm{OPh}, 4-\mathrm{CF}_{3} \mathrm{Ph}$

Scheme. Synthesis of $\left[\left(8-R_{1}-9-R_{2}-10-R_{3}-3-R-2-o x o-2 H-[1,2,4]\right.\right.$ triazino[2,3-c]-quinazoline-6-yl)thio]acetic acids and the functional derivatives.

pounds was determined by the absence of visual growth in the test tube with the minimal concentration of the substance, the minimal bactericidal/fungicidal concentration was determined by the absence of growth on agar after inoculation of the microorganism from transparent test-tubes. Dimethylsulfoxide was used as a solvent, the initial solution concentration was $1 \mathrm{mg} / \mathrm{ml}$. For preliminary screening the standard test cultures such as Staphylococcus aureus ATCC 25923, Escherichia coli ATCC 25922, Pseudomonas aeruginosa ATCC 27853 and Candida albicans ATCC 885-653 were used. All test strains were received from the bacteriological laboratory at the Zaporizhzhya Regional Laboratory Centre of the State Sanitary and Epidemiological Service of Ukraine. Nitrofual, trimetoprim and ketoconazol were used as reference compounds with the proved antibacterial/antifungal activity. Additional quality control of the culture medium and solvents was conducted by the methods commonly used.

\section{Results and Discussion}

\section{Chemistry}

As initial compounds, 3-R-6-thio-6,7-dihydro- $2 \mathrm{H}$ $[1,2,4]$ triazino[2,3-c]-quinazolin-2-ones (1) and their potassium salts were used; the salts were obtained according to the known protocols of 6-R-3-(3- $\mathrm{R}_{1}-4-\mathrm{R}_{2}-5-\mathrm{R}_{3}-2-$ amnophenyl)-1,2,4-triazin-5(2H)-ones with carbon disulfide, potassium hydroxide in the ethyl alcohol medium and potassium ethylxanthate in propanol-2 $[7,8]$.

The synthesis of $\left[\left(8-\mathrm{R}_{1}-9-\mathrm{R}_{2}-10-\mathrm{R}_{3}-3-\mathrm{R}-2-\mathrm{oxo}-2 \mathrm{H}-\right.\right.$ $[1,2,4]$ triazino[2,3-c]quinazolin-6-yl)thio]acetic acids (3) was conducted via alkylation of potassium thiolates with chloroacetic acids in propanol-2 or propanol-2 - water in the presence of the equivalent amount of sodium hydroxide (Scheme). After acidification of the reaction mixture ( $\mathrm{pH} 3-4)$, the solids of the corresponding acids were formed with high yields. Amides 4 were synthesized by alkylation of potassium thiolates $\mathbf{2}$ with chloracetamide or $\mathrm{N}-\mathrm{R}_{4}$-chloracetamides in propanol-2. Unfortunately, we failed to obtain amides 4 by amonolysis of the corresponding ester because of low reactivity of the compounds mentioned.

Synthesis of nitriles 5 by dehydratation of the proper amides 4 with phosphorous-oxychloride in dichlormethane was not successful in all cases. The fact mentioned was caused by low yields and problems with isolation of the target compound from the reaction mixture. Thus, nitriles 5 were synthesized by interaction of potassium thiolates with chloroacetonitrile in propanol-2.

We noted that thions $\mathbf{1}$ were also alkylated by chloroacetic acid and their derivatives in propanol-2 in the presence of sodium hydroxide. The reaction easily flows for 1-1.5 h., elongation of the process did not lead to the increase of the yields.

The purity of the compounds synthesized was confirmed by LC-MS, the structure by elemental analysis, ${ }^{1} \mathrm{H}$ and ${ }^{13} \mathrm{C}$ NMR-spectrometry.

In LC-MS spectra of compounds $\mathbf{3 , 4}$ and $\mathbf{5}$ in most cases the positive ions $[\mathrm{M}+1]$ and $[\mathrm{M}+3]$ were observed, the $\mathrm{m} / \mathrm{z}$ values of the ions mentioned corresponded to the molecular weight of the target compounds. In LC-MS spectra acids 3.2-3.4 we have found the signals, which according to their $\mathrm{m} / \mathrm{z}$ value belong to the fragmentary ion $\left[\mathrm{M}-\mathrm{CH}_{2} \mathrm{COOH}\right]^{+}$, and it additionally confirms the structure of the acids synthesized.

In ${ }^{1} \mathrm{H} N M R$-spectra of compounds $\mathbf{3}, \mathbf{4}$ and the singlet signal of the $\mathrm{SCH}_{2}$-group at 4.33-3.89 ppm, the chemical shift of the signal was caused by the nature of the substituent at the carbonyl group. The signal of the proton mentioned was observed in the lower field of ${ }^{1} \mathrm{H}-\mathrm{NMR}$ of the corresponding nitriles 5 (4.33-4.32 ppm). The pro- 
The antimicrobial and antifungal activity of the compounds synthesized

\begin{tabular}{|c|c|c|c|c|c|c|c|c|c|c|c|c|}
\hline \multirow{2}{*}{$\begin{array}{c}\text { Comp. } \\
\text { No. }\end{array}$} & \multirow[b]{2}{*}{$\mathrm{R}^{*}$} & \multirow[b]{2}{*}{$\mathrm{R}_{1}$} & \multirow[b]{2}{*}{$\mathrm{R}_{2}$} & \multirow[b]{2}{*}{$\mathrm{R}_{3}$} & \multicolumn{2}{|c|}{ E. coli } & \multicolumn{2}{|c|}{ S. aureus } & \multicolumn{2}{|c|}{ P. aerugenosa } & \multicolumn{2}{|c|}{ C. albicans } \\
\hline & & & & & $\begin{array}{c}\mathrm{MIC} \\
\mu \mathrm{g} / \mathrm{ml}\end{array}$ & $\begin{array}{c}\mathrm{MBC}, \\
\mu \mathrm{g} / \mathrm{ml}\end{array}$ & $\begin{array}{c}\mathrm{MIC} \\
\mu \mathrm{g} / \mathrm{ml}\end{array}$ & $\begin{array}{l}\mathrm{MBC}, \\
\mu \mathrm{g} / \mathrm{ml}\end{array}$ & $\begin{array}{l}\mathrm{MIC} \\
\mu \mathrm{g} / \mathrm{ml}\end{array}$ & $\begin{array}{c}\mathrm{MBC} \\
\mu \mathrm{g} / \mathrm{ml}\end{array}$ & $\begin{array}{c}\mathrm{MIC} \\
\mu \mathrm{g} / \mathrm{ml}\end{array}$ & $\begin{array}{l}\mathrm{MFC}, \\
\mu \mathrm{g} / \mathrm{ml}\end{array}$ \\
\hline 3.3 & 4-(Me) $\mathrm{C}_{6} \mathrm{H}_{4}$ & $\mathrm{H}$ & $\mathrm{H}$ & $\mathrm{H}$ & 100 & 100 & 25 & 100 & 50 & 100 & 50 & 50 \\
\hline 3.4 & 3,4-(Me ${ }_{2} \mathrm{C}_{6} \mathrm{H}_{3}$ & $\mathrm{H}$ & $\mathrm{H}$ & $\mathrm{H}$ & 100 & 200 & 12.5 & 100 & 50 & 100 & 50 & 100 \\
\hline 3.5 & 4-(Et) $\mathrm{C}_{6} \mathrm{H}_{4}$ & $\mathrm{H}$ & $\mathrm{H}$ & $\mathrm{H}$ & 100 & 200 & 12.5 & 50 & 100 & 200 & 50 & 100 \\
\hline 3.6 & 4-(i-Pr) $-\mathrm{C}_{6} \mathrm{H}_{4}$ & $\mathrm{H}$ & $\mathrm{H}$ & $\mathrm{H}$ & 100 & 200 & 12.5 & 25 & 100 & 100 & 50 & 100 \\
\hline 3.14 & $4-\mathrm{F}-\mathrm{C}_{6} \mathrm{H}_{4}$ & $\mathrm{H}$ & $\mathrm{H}$ & $\mathrm{Br}$ & 200 & 200 & 12.5 & 50 & 100 & 200 & 100 & 100 \\
\hline 4.1 & $\mathrm{Me}$ & $\mathrm{H}$ & $\mathrm{H}$ & $\mathrm{H}$ & 50 & 100 & 100 & 200 & 200 & 200 & 25 & 100 \\
\hline 4.5 & 4-(Et) $\mathrm{C}_{6} \mathrm{H}_{4}$ & $\mathrm{H}$ & $\mathrm{H}$ & $\mathrm{H}$ & 100 & 100 & 25 & 100 & 100 & 200 & 50 & 100 \\
\hline 4.6 & 4-(tert-Bu) $\mathrm{C}_{6} \mathrm{H}_{4}$ & $\mathrm{H}$ & $\mathrm{H}$ & $\mathrm{H}$ & 100 & 200 & 25 & 100 & 100 & 200 & 50 & 100 \\
\hline 4.7 & 4- $(\mathrm{MeO}) \mathrm{C}_{6} \mathrm{H}_{4}$ & $\mathrm{H}$ & $\mathrm{H}$ & $\mathrm{H}$ & 100 & 200 & 25 & 25 & 100 & 200 & 25 & 50 \\
\hline 4.8 & 4-(EtO) $\mathrm{C}_{6} \mathrm{H}_{5}$ & $\mathrm{H}$ & $\mathrm{H}$ & $\mathrm{H}$ & 100 & 100 & 12.5 & 50 & 100 & 200 & 50 & 100 \\
\hline 4.11 & $\mathrm{Ph}$ & $\mathrm{Me}$ & $\mathrm{H}$ & $\mathrm{H}$ & 100 & 100 & 50 & 100 & 50 & 100 & 25 & 100 \\
\hline 4.12 & $4-\mathrm{F}-\mathrm{C}_{6} \mathrm{H}_{4}$ & $\mathrm{Br}$ & $\mathrm{H}$ & $\mathrm{H}$ & 100 & 100 & 50 & 200 & 100 & 200 & 25 & 50 \\
\hline 4.13 & $\mathrm{Ph}$ & $\mathrm{H}$ & $\mathrm{F}$ & $\mathrm{H}$ & 100 & 200 & 12.5 & 100 & 100 & 200 & 12.5 & 12.5 \\
\hline 4.14 & 4- $(\mathrm{MeO}) \mathrm{C}_{6} \mathrm{H}_{4}$ & $\mathrm{H}$ & $\mathrm{F}$ & $\mathrm{H}$ & 200 & 200 & 12.5 & 25 & 200 & 200 & 12.5 & 25 \\
\hline 4.16 & 4- $\mathrm{FC}_{6} \mathrm{H}_{5}$ & $\mathrm{H}$ & $\mathrm{F}$ & $\mathrm{H}$ & 100 & 200 & 25 & 50 & 200 & 200 & 25 & 50 \\
\hline 4.26 & 4-F- $\mathrm{C}_{6} \mathrm{H}_{4}$ & $\mathrm{H}$ & $\mathrm{H}$ & $\mathrm{Br}$ & 50 & 100 & 100 & 200 & 200 & 200 & 25 & 100 \\
\hline 5.10 & $\mathrm{Ph}$ & $\mathrm{H}$ & $\mathrm{F}$ & $\mathrm{H}$ & 100 & 200 & 50 & 100 & 100 & 200 & 12.5 & 25 \\
\hline 5.11 & 4-(MeO) $\mathrm{C}_{6} \mathrm{H}_{4}$ & $\mathrm{H}$ & $\mathrm{F}$ & $\mathrm{H}$ & 100 & 200 & 50 & 100 & 100 & 200 & 25 & 50 \\
\hline 5.13 & $\mathrm{Ph}$ & $\mathrm{H}$ & $\mathrm{H}$ & $\mathrm{Cl}$ & 25 & 100 & 100 & 200 & 50 & 100 & 50 & 100 \\
\hline 5.14 & 4- $\mathrm{MeC}_{6} \mathrm{H}_{4}$ & $\mathrm{H}$ & $\mathrm{H}$ & $\mathrm{Cl}$ & 25 & 100 & 100 & 200 & 50 & 100 & 50 & 100 \\
\hline \multicolumn{5}{|c|}{ Nitrofural } & 1.5 & - & 6.25 & - & 6.25 & - & 25.0 & - \\
\hline \multicolumn{5}{|c|}{ Trimethoprim } & 50 & 50 & 31.2 & 62.5 & 62.5 & 125 & 62.5 & 125 \\
\hline \multicolumn{5}{|c|}{ Ketoconazole } & - & - & - & - & - & - & 25 & - \\
\hline
\end{tabular}

* - compounds 3.1, 3.2, 3.7-3.13, 4.2-4.4, 4.9, 4.10, 4.15, 4.17-4.25, 5.1-5.9, 5.15-5.19 exhibit the antibacterial activity $\leq 50 \mu \mathrm{g} / \mathrm{ml}$.

ton of the carboxylic group of acids was observed at 13.92-12.90 ppm. Protons of the primary amide group of compounds 4 as result of the $\mathrm{C}=\mathrm{O}$ group effect were observed as two non-equivalent one-proton singlets at 7.30-7.08 ppm and 7.58-7.53 ppm. For the secondary amides 4 the amide protons were observed as a singlet at 10.59-10.00 ppm (for 4.9, 4.16, 4.24, 4.25) or 8.828.5 ppm (4.19, 4.21, 4.22). Compounds (4.19, 4.21, 4.22) were also characterized by a two-proton doublet of the $\mathrm{NCH}_{3}$ fragment in ${ }^{1} \mathrm{HNMR}$ spectra. Aromatic protons of the non-substituted triazinoquinazoline cycle of compounds $3,4,5$ formed systems with two one-proton doublets $\mathrm{H}-8$ and $\mathrm{H}-11$ and two one-proton triplets H-9 and $\mathrm{H}-10$. Signals of aromatic protons of the para-substituted phenyl moiety in position 3 of compounds 3,4 and $\mathbf{5}$ were observed as the $\mathrm{A}_{2} \mathrm{~B}_{2}$-system consisting of two-proton doublets (H-3, H-5 and H-2, H-6), at the same time, signals of the unsubstituted one were observed as a multiplet (H-3, H-4, H-5) and a two-proton doublet.

Signals of $s p^{3}$-hybrid carbons belonging to $\mathrm{CH}_{3}$ (18.19 ppm), $\mathrm{SCH}_{2}$ (34.21-38.94 ppm), $\mathrm{CH}_{3} \mathrm{O}$ (55.94 ppm) were observed in the high field of ${ }^{13} \mathrm{C}$ NMR-spectra of some compounds. According to ${ }^{13} \mathrm{C}$ NMR spectra the most deshielded carbons were located in the - $\mathrm{COOH}$-group (170.04-170.08 ppm) and positions 2 and 6 of $[1,2,4]$ triazino[2,3-c]quinazoline system.

\section{Antimicrobial and antifungal activities}

The antimicrobial assay has shown that the compounds synthesized exhibit the antibacterial and antifungal activity against the strains studied. Thus, 2-[(3methyl-2-oxo- $2 H$ - $[1,2,4]$ triazino[2,3-c]quinazoline-6yl)thio]acetic acids (3.1, MIC 50-200 $\mu \mathrm{g} / \mathrm{ml})$ exhibit a moderate action against E.coli, $S$. aureus and P. aerugino$s a$, but a high activity against $C$. albicans (MIK $50 \mu \mathrm{g} / \mathrm{ml}$, Table). Chemical modification of compound 3.1 via changing the methyl group in position 3 into the phenyl moiety (3.2) did not lead to the activity increase. Introduction of fluorine $(3.9)$ and alkoxy groups $(3.7,3.8)$ in the phenyl moiety also did not lead to the increase of the antibacterial and antifungal activity. At the same time 4-alkylphenyl derivatives (3.3-3.6) exhibit a high inhibiting effect against $S$. aureus (MIC $12.5-25 \mu \mathrm{g} / \mathrm{ml}$ ). We noticed that the increase in activity was observed in case of introduction of the additional methyl group (compound 3.4), elongation (3.5) and branching (3.6) of the alkyl group in the phenyl substituent (Table). Introduction of the addition substituent (fluoro-, chloro-, bromoand methyl group) in position 8, 9, 10 did not cause essential changes of the antimicrobial and antifungal activity. Only compound 3.14 containing bromine in position 10 and (4-fluoro)phenyl in position 3 exhibits a high activity against $S$. aureus (MIC $12.5 \mu \mathrm{g} / \mathrm{ml}$ ). 
Following modification of the carboxylic group (compounds 3.1-3.14) in the amide fragment (4.1-4.26) resulted in increasing the antibacterial and antifungal activity against the strains studied. We noticed that some amides (4.1, 4.3, 4.4, 4.9, 4.15, 4.17. 4.18, 4.20, 4.21, 4.26) unlike acids (3.1-3.15) exhibited the antibacterial activity against $E$. coli (MIC $50 \mu \mathrm{g} / \mathrm{ml}$ ) at the same level as trimetoprim (MIC $50 \mu \mathrm{g} / \mathrm{ml}$ ). Moreover, amides 4.1-4.26 showed a high antifungal action against $C$. albicans (MIC 12.5-50 $\mu \mathrm{g} / \mathrm{ml}$ ) exceeding the activity of trimetoprim (MIC $62.5 \mu \mathrm{g} / \mathrm{ml}$ ) and was comparable to nitrofural (MIC $25.0 \mu \mathrm{g} / \mathrm{ml}$, compounds 4.1, 4.7, 4.11, $4.14,4.16,4.26$ ). A considerable attention as to antifungal agents should be paid to compounds $\mathbf{4 . 1 3}$ and $\mathbf{4 . 1 4}$ (MIC $12.5 \mu \mathrm{g} / \mathrm{ml}$ ) exceeding the activity of ketocona$\mathrm{zol}(\mathrm{MIC} 25 \mu \mathrm{g} / \mathrm{ml}$ ).

We noted that as in case of acids amides 4.1-4.26 were more active against $S$. aureus (MIC 12.5-100 $\mu \mathrm{g} / \mathrm{ml}$ ). The modification of compound $\mathbf{4 . 1}$ was conducted via changing the methyl group in position 3 of the triazinoquinazoline system into phenyl (4.2), 4-alkylphenyl (4.3, 4.5-4.6), 3,4-dimethylphenyl (4.4) and 4-alkoxyphenil $(4.7,4.8)$ results in the activity increase. Unlike acids $(3.11,3.12)$, amides $(4.13-4.16)$ containing a fluorine atom in position 9 were more active against $S$. aureus. Substitution of fluorine (4.13-4.16) into bromine (4.17) caused insignificant decrease in activity (MIC 50 $\mu \mathrm{g} / \mathrm{ml}$ ). The complete loss of activity was observed in case of translocation of bromine from 9 to 10 position (compounds 4.22, 4.23, 4.26; MIC 50-100 $\mu \mathrm{g} / \mathrm{ml}$ ) and introduction of chlorine (4.19 MIC $50 \mu \mathrm{g} / \mathrm{ml})$. Introduction of the $\mathrm{N}$-substituted amide group (compounds $4.9,4.15,4.18,4.20,4.21,4.25,4.26)$ irrespective of the substituent and its location in the molecule did not affect or cause insignificant decrease of action against S. aureus.

Nitriles (5.1-5.19) unlike acids (3.1-3.15) and amides (4.1-4.26) were active against $P$. aeruginosa (MIC
$50-100 \mu \mathrm{g} / \mathrm{ml}$ ). Among all the compounds studied only compounds 5.13 and $\mathbf{5 . 1 4}$ revealed a high antimicrobial action against $E$. coli (MIC $25 \mu \mathrm{g} / \mathrm{ml}$ ). At the same time the inhibitory activity of nitriles (5.1-5.19) against $S$. aureus comparing to acids and amides were insignificant (MIC 50-200 $\mu \mathrm{g} / \mathrm{ml})$. Nitriles (5.1-5.19) also exhibited a high inhibitory action against $C$. albicans (MIC 12.5-100 $\mu \mathrm{g} / \mathrm{ml}$ ). Among the compounds studied our attention was focused on compound $\mathbf{5 . 1 0}$ (MIC $12.5 \mu \mathrm{g} / \mathrm{ml}$ ) exceeding ketoconazole (MIC $25 \mu \mathrm{g} / \mathrm{ml}$ ) in its activity.

Thus, the antimicrobial and antifungal activity of 2-[8- $\mathrm{R}_{1}-9-\mathrm{R}_{2}-10-\mathrm{R}_{3}-3-\mathrm{R}-2-$ oxo-2 $H$-[1,2,4]triazino[2,3-c] quinazoline-6-yl)thio]acetic acids studied and their derivatives was caused by the nature of substituents in position 6 , substituents of the phenyl moiety in position and substituents in positions $8,9,10$ of the triazinoquinazoline system. We have found that the presence of 4-alkylphenyl (compounds 3.3-3.6, 4.3-4.6; MIC 12.5$25 \mu \mathrm{g} / \mathrm{ml}), 4$-alkoxyphenyl (4.7, 4.8; MIC $12.5-50 \mu \mathrm{g} / \mathrm{ml})$ substituents in position 3 and fluorine in position 9 4.134.16; MIC $12.5-25 \mu \mathrm{g} / \mathrm{ml}$ ) contributes positively to the high activity against $S$. aureus, the presence of fluorine in position 9 also promotes the activity against $C$. albicans $(4.13,4.14$; MIC $12.5 \mu \mathrm{g} / \mathrm{ml})$.

\section{CONCLUSIONS}

In the present paper 50 new derivatives of 2-[8- $\mathrm{R}_{1}$ 9- $\mathrm{R}_{2}-10-\mathrm{R}_{3}-3-\mathrm{R}-2-$ oxo- $2 H$ - $[1,2,4]$ triazino[2,3-c] quinazoline-6-yl)thio]acetic acids have been described. The compounds synthesized have been tested for the antimicrobial and antifungal activity using standard test cultures: Staphylococcus aureus ATCC 25923, Escherichia coli ATCC 25922, Pseudomonas aeruginosa ATCC 27853 and Candida albicans ATCC 885-653. It has been shown that the compounds synthesized exhibit a high antimicrobial activity against St. aureus (compounds 3.3-3.6, 4.34.6, 4.7, 4.8, 4.13-4.16; MIC $12,5-25 \mu \mathrm{g} / \mathrm{ml})$ and C. albicans (compounds 4.13, 4.14; MIC 12,5 $\mu \mathrm{g} / \mathrm{ml}$ ). The "structure-activity" relationship has been discussed.

\section{REFERENCES}

1. Antipenko L.N., Karpenko A.V., Kovalenko S.I. et al. // Arch. Pharm. - 2009. - Vol. 342, Iss. 11. - P. 651-662.

2. Antipenko L., Karpenko A., Kovalenko S. et al. // Chem. Pharm. Bull. (Tokyo). - 2009. - Vol. 57, №6. - P. 580-585.

3. Available from: http://www.drugbank.ca/structures/search/.

4. Baruah B., Dasu K., Vaitilingam B. et al. // Bioorg. Med. Chem. - 2004. - Vol. 12, Iss. 9. - P. 1991-1994.

5. Bedi P.M.S., Kumar V., Mahajan M.P. // Bioorg. Med. Chem. Lett. - 2004. - Vol. 14, Iss. 20. - P. 5211-5213.

6. Berest G.G., Kovalenko S.I., Nosulenko I.S. et al. // Sci. Pharm. - 2012. - Vol. 80, Iss. 1. - P. 37-65.

7. Berest G.G., Kovalenko S.I., Sinyak R.S. et al. // J. of Org. and Pharmac. Chem. - 2010. - №8. - P. 42-52.

8. Berest G.G., Voskoboynik O.Y., Kovalenko S.I. et al. // Eur. J. Med. Chem. - 2011. - Vol. 46, Iss. 12. - P. 6066-6074.

9. Institute CaLS. Performance Standards for Antimicrobial Disk Susceptibility Tests; Approved Standard.

10. Jantová S., Ovádeková R., Letašiová S. et al. // Folia Microbiol. (Praha). - 2005. - Vol. 50, Iss. 2. - P. 90-94.

11. Kovalenko S., Nosulenko I., Voskoboynik A. et al. // Med. Chem. Res. - 2013. - Vol. 22, Iss. 6. - P. 2610-2632.

12. Kovalenko S.I., Voskoboynik A.Yu., Berest G.G. et al. // Sci. Pharm. - 2012. - Vol. 80, Iss. 4. - P. 837-865.

13. Kuarm B.S., Reddy Y.T., Madhav J.V. et al. // Bioorg. Med. Chem. Lett. - 2011. - Vol. 21, Iss. 1. - P. 524-527.

14. Lamazzi C., Léonce S., Pfeiffer B. et al. // Bioorg. Med. Chem. Lett. - 2000. - Vol. 10, Iss. 19. - P. 2183-2185.

15. Nasr M.N., Gineinah M.M., El-Bendary E.R. // Arch. Pharm. - 2003. - Vol. 336, Iss. 12. - P. 560-566. 
16. Ovadekova R.J.S., Theiszova M., Labuda I. // J. Biomed. Pap. Med. Fac. Univ. Palacky Olomouc Czech. Repub. - 2005. - Vol. 149, Iss. 2. - P. 455-459.

17. Rohini R., Muralidhar Reddy P., Shanker K. et al. // Eur. J. Med. Chem. - 2010. - Vol. 45, Iss. 3. - P. 1200-1205.

18. Rohini R., Shanker K., Reddy P.M. et al. // Eur. J. Med. Chem. - 2009. - Vol. 44, Iss. 8. - P. 3330-3339.

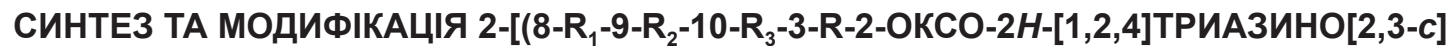 ХІНАЗОЛІН-6-ІЛ)ТІО]ОЦТОВИХ КИСЛОТ, СПРЯМОВАНІ НА ПОШУК СПОЛУК 3 АНТИБАКТЕРІАЛЬНОЮ ТА ПРОТИГРИБКОВОЮ ДІЄЮ І.С.Носуленко, О.Ю.Воскобойнік, Г.Г.Берест, С.І.Коваленко, О.М.Камишний, Н.М.Поліщук \\ Ключові слова: синтез; хіназоліни; триазини; антибактеріальна активність;} протигрибкова активність

В представленій роботі описано синтез 50 нових похідних 2-[8- $R_{1}-9-R_{2}-10-R_{3}-3-R-2-о к с о-2 H-$ [1,2,4]триазино[2,3-с]хіназолін-6-іл)тіо]оцтових кислот. Показано, що алкілування калій 8- $R_{1-}$

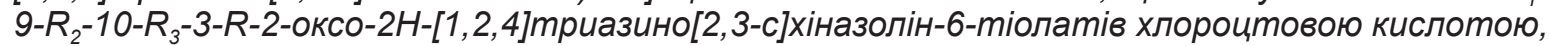
хлорацетамідом, $N$ - $R_{4}$-хлорацетамідами та хлорацетонітрилом веде до утворення відповід-

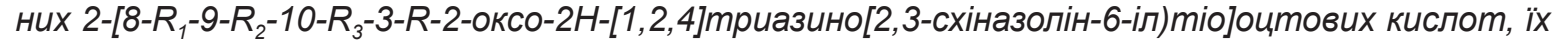
амідів та нітрилів. Для відповідних кислот та нітрилів були опрацьовані альтернативні синтетичні підходи. Також були обговорені обмеження у синтезі цільових сполук. Так, було по-

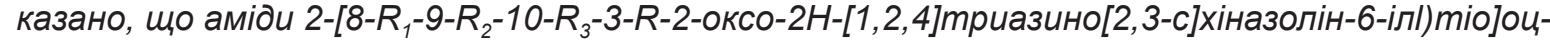
тових кислот не можуть бути одержані амонолізом відповідних естерів внаслідок низької реакційної здатності останніх. Заявлено, що синтез нітрилів дегідратацією відповідних амідів хлорокисом фросфрору у дихлорометані був успішним не в усіх випадках. Зазначений фракт обумовлено вкрай низькими виходами цільових сполук, що пов'язано зі значними проблемами при їх виділенні з реакційної суміші. Структуру синтезованих сполук було визначено за допомогою комплексу сучасних фрізико-хімічних методів $\left({ }^{1} \mathrm{H},{ }^{13} \mathrm{C} N \mathrm{NMR}\right.$, LC-MS-спектрами). Синтезовані сполуки були випробувані на антимікробну та протигрибкову дію з використанням стандартних тест-культур: Staphylococcus aureus ATCC 25923, Escherichia coli ATCC 25922, Pseudomonas aeruginosa ATCC 27853 ma Candida albicans ATCC 885-653. Показано, що сполуки 3.3-3.6, 4.3-4.6, 4.7, 4.8, 4.13-4.16 проявляють виражену активність по відношенню до St. aureus (MIC 12,5-25 $\mu \mathrm{g} / \mathrm{ml}$ ), а сполуки 4.13, 4.14 також по відношенню до C. albicans (MIC 12,5 $\mu \mathrm{g} / \mathrm{ml}$ ). В рамках статті обговорено взаємозв'язок «структура-біологічна дія».

\section{СИНТЕЗ И МОДИФИКАЦИЯ 2-[(8-R $\mathbf{R}_{1}-9-\mathrm{R}_{2}-10-\mathrm{R}_{3}-3-\mathrm{R}-2-\mathrm{OКСО-2H-[1,2,4]ТРИАЗИНО[2,3-c]}$ ХИНАЗОЛИН-6-ИЛ)ТИО]УКСУСНЫХ КИСЛОТ, НАПРАВЛЕННЫЕ НА ПОИСК СОЕДИНЕНИЙ С АНТИБАКТЕРИАЛЬНЫМ И ПРОТИВОГРИБКОВЫМ ДЕЙСТВИЕМ И.С.Носуленко, А.Ю.Воскобойник, Г.Г.Берест, С.И.Коваленко, А.М.Камышный, Н.М.Полищук}

Ключевые слова: синтез; хиназолины; триазины; антибактериальная активность; противогрибковая активность

В представленной работе описан синтез 50 новых производных 2-[8- $R_{1}-9-R_{2}-10-R_{3}-3-R-2-о к с о-$ $2 H-[1,2,4]$ тиазино[2,3-с]хиназолин-6-ил)тио]уксусных кислот. Показано, что алкилирование

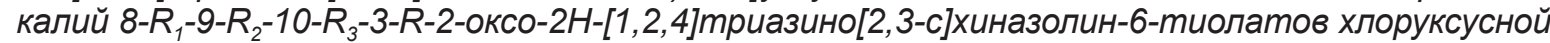
кислотой, хлорацетамидом, $N$ - $R_{4}$-хлорацетамидами и хлорацетонитрилом приводит к об-

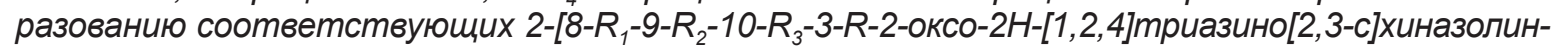
6-ил)тио]уксусных кислот, их амидов и нитрилов. Для соответствующих кислот и нитрилов были разработаны альтернативные синтетические подходы. Также были обговорены ограничения в синтезе целевых соединений. Так было показано, что амиды 2-[8- $R_{1}-9-R_{2}-10-$ $R_{3}$-3-R-2-оксо-2H-[1,2, 4]триазино[2,3-с]хиназолин-6-ил)тио]уксусных кислот не могут быть получены амонолизом соответствующих эфриров ввиду низкой реакционной активности последних. Заявлено, что синтез нитрилов дегидратацией соответствующих амидов хлорокисью фросфора в дихлорметане был успешен не во всех случаях. Данный фракт был обусловлен крайне низкими выходами целевых соединений вследствие значительных проблем при их выделении из реакционных смесей. Структура синтезированных соединений подтверждена с помощью современных фризико-химических методов $\left({ }^{1} \mathrm{H},{ }^{13} \mathrm{C}\right.$ NMR, LC-MS-спектры). Синтезированные соединения были исследованы на противомикробную и противогрибковую активность с использованием стандартных тест культур: Staphylococcus aureus ATCC 25923, Escherichia coli ATCC 25922, Pseudomonas aeruginosa ATCC 27853 u Candida albicans АТСС 885-653. Показано, что соединения 3.3-3.6, 4.3-4.6, 4.7, 4.8, 4.13-4.16 проявляют выраженную активность по отношению к St. aureus (MIC 12,5-25 $\mu \mathrm{g} / \mathrm{ml}$ ), а соединения 4.13, 4.14 также по отношению к C. albicans (MIC 12,5 $\mu \mathrm{g} / \mathrm{ml}$ ). Также в рамках статьи обсуждена взаимосвязь «структура-биологическое действие». 